\title{
Multigenome DNA sequence conservation identifies Hox cis-regulatory elements
}

\author{
Steven G. Kuntz, ${ }^{1,2}$ Erich M. Schwarz, ${ }^{1}$ John A. DeModena, ${ }^{1,2}$ Tristan De Buysscher, ${ }^{1}$ \\ Diane Trout, ${ }^{1}$ Hiroaki Shizuya, ${ }^{1}$ Paul W. Sternberg, ${ }^{1,2,3}$ and Barbara J. Wold ${ }^{1,3}$ \\ ${ }^{1}$ Division of Biology, California Institute of Technology, Pasadena, California 91125, USA; ${ }^{2}$ Howard Hughes Medical Institute, \\ California Institute of Technology, Pasadena, California 91125, USA
}

\begin{abstract}
To learn how well ungapped sequence comparisons of multiple species can predict cis-regulatory elements in Caenorhabditis elegans, we made such predictions across the large, complex ceh-13/lin-39 locus and tested them transgenically. We also examined how prediction quality varied with different genomes and parameters in our comparisons. Specifically, we sequenced $\sim 0.5 \%$ of the $C$. brenneri and $C$. sp. 3 PSIOIO genomes, and compared five Caenorhabditis genomes ( $C$. elegans, C. briggsae, $C$. brenneri, $C$. remanei, and C. sp. 3 PSIOIO) to find regulatory elements in $22.8 \mathrm{~kb}$ of noncoding sequence from the ceh-13/lin-39 Hox subcluster. We developed the MUSSA program to find ungapped DNA sequences with $\mathrm{N}$-way transitive conservation, applied it to the ceh-13/lin-39 locus, and transgenically assayed 21 regions with both high and low degrees of conservation. This identified 10 functional regulatory elements whose activities matched known ceh-13/lin-39 expression, with $100 \%$ specificity and a $77 \%$ recovery rate. One element was so well conserved that a similar mouse Hox cluster sequence recapitulated the native nematode expression pattern when tested in worms. Our findings suggest that ungapped sequence comparisons can predict regulatory elements genome-wide.
\end{abstract}

[Supplemental material is available online at www.genome.org. The sequence data from this study have been submitted to GenBank (http://www.ncbi.nlm.nih.gov/Genbank/) under accession nos. FJ362353-F]36238.]

Despite knowledge of entire genome sequences, discovering cisregulatory DNA elements remains surprisingly inefficient. In animal genomes, cis-regulatory elements are located unpredictably around or within the genes they regulate (Woolfe et al. 2005; Davidson 2006; Pennacchio et al. 2006; Engström et al. 2007). These elements, when dissected further, often prove to be composed of individual transcription factor binding sites that are often very loosely defined (Sandelin et al. 2004). Transgenic analysis in vivo is the most definitive way to show that a sequence is regulatory, but it is also the most time consuming and expensive. It is therefore desirable to use other criteria, such as preferential sequence conservation, to identify regions most likely to be functional. To evaluate a strategy for phylogenetic footprinting using four other Caenorhabditis species, we dissected the cis-regulatory structure of a Hox cluster in the nematode Caenorhabditis elegans (Fig. 1A).

If two or more species are evolutionarily close enough to show common development and physiology, their genomes are expected to share an underlying gene regulatory network driven by cis-regulatory elements with conserved sequences of several hundred base pairs (Tagle et al. 1988; Davidson 2006; Brown et al. 2007; Li et al. 2007). Within a functional cis-regulatory element, individual transcription-factor binding sites are generally short ( 6-20 bp) with statistical preferences, not strict requirements, for specific bases (Sandelin et al. 2004). Statistical overrepresentation of such motifs has been useful for identifying transcription-factor binding sites common to coregulated genes

\footnotetext{
${ }^{3}$ Corresponding authors.
}

E-mail woldb@caltech.edu; fax (626) 395-5750.

E-mail pws@caltech.edu; fax (626) 568-8012.

Article published online before print. Article and publication date are at http:// www.genome.org/cgi/doi/10.1101/gr.085472.108. in C. elegans (Ao et al. 2004; Gaudet et al. 2004; Wenick and Hobert 2004; Pauli et al. 2006; Etchberger et al. 2007; McGhee et al. 2007; Zhao et al. 2007). However, this approach requires a known set of coregulated genes, a limitation that cross-species genomic comparison methods do not have. The simplest genomic comparison method is all-against-all matching of ungapped sequence windows, which is well suited for finding cis-regulatory elements under selective pressure against insertions and deletions (Brown et al. 2002; Cameron et al. 2005). This kind of comparison reveals orientation-independent, one-to-many, and many-to-many relationships, all of which are possible for conserved cis-regulatory sequences, yet invisible in standard global alignments. While ungapped comparisons can highlight regulatory regions, they are not expected to resolve individual transcription-factor binding sites within them. However, different prediction biases from sequence conservation versus statistical over-representation can complement one another (Wang and Stormo 2003; Bigelow et al. 2004; Tompa et al. 2005; Chen et al. 2006).

Since purely random pairing of unrelated 100-bp DNA segments typically yields two perfect 6-bp matches (Dickinson 1991), comparing three or more species should identify sequences under selective pressure with greater accuracy than comparing only two (Boffelli et al. 2004; Sinha et al. 2004; Eddy 2005; Stone et al. 2005). This has recently been done for budding yeasts (Cliften et al. 2003; Kellis et al. 2003), Drosophila (Stark et al. 2007), and vertebrates (Krek et al. 2005; Xie et al. 2005, 2007; Pennacchio et al. 2006; McGaughey et al. 2008). Vertebrates have many conserved sequences that may be regulatory, but most have unknown functions (Bejerano et al. 2004; Boffelli et al. 2004; Ovcharenko et al. 2005; Ahituv et al. 2007) that are difficult to test in all cell types throughout the life cycle, especially in mammals. 
A

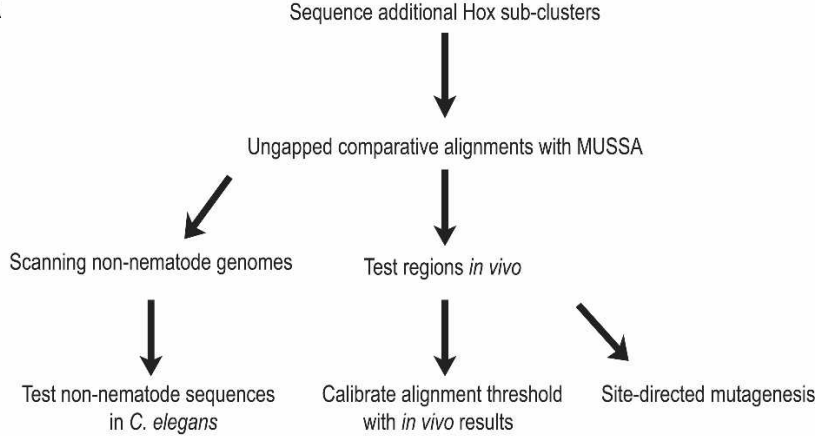

B

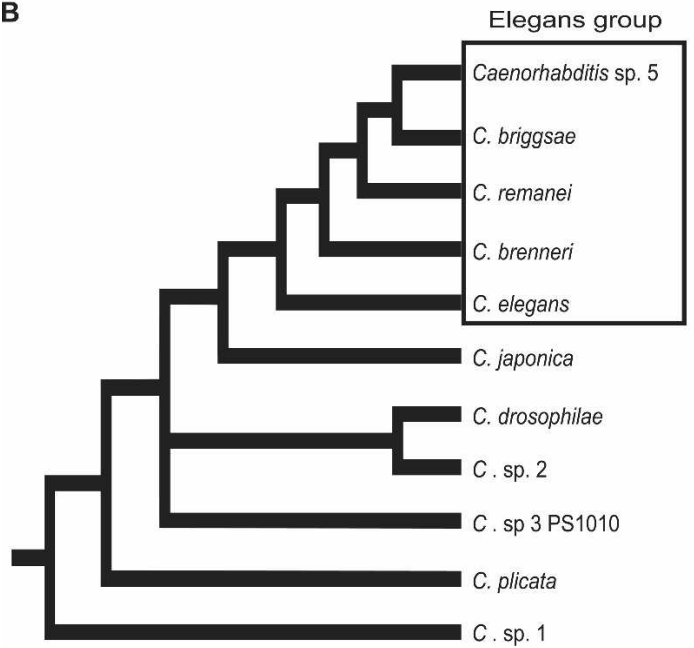

Figure 1. Experimental flow and Caenorhabditis phylogeny. (A) The experimental rationale of the project is shown. (B) Phylogeny of nematodes within the Caenorhabditis genus from Kiontke et al. (2007). The Elegans group and C. sp. 3 PS1010 are dealt with in this study.

The nematode Caenorhabditis elegans has a compact genome (100 Mb, 27,000 genes) and body ( $\sim 1000$ somatic cells in adults), which should allow candidate regulatory elements to be tested for function throughout development and across all cell types (Sulston and Horvitz 1977; Kimble and Hirsh 1979; Hillier et al. 2005). Although C. elegans is the most familiar Caenorhabditis species, others are available for multispecies genomic comparisons (Fig. 1B) (Sudhaus and Kiontke 1996, 2007; Baldwin et al. 1997; Stothard and Pilgrim 2006). Sibling species (the Elegans group, including C. brenneri) are difficult to distinguish from $C$. elegans morphologically, save for sex differences (Sudhaus and Kiontke 1996; Kiontke et al. 2004). C. japonica, the closest outgroup, shows some morphological differences, but they are relatively minor (Kiontke et al. 2002), while the more distant $C$. sp. 3 PS1010 has distinct morphology and behavior (Sudhaus and Kiontke 1996; Cho et al. 2004; Kiontke et al. 2004). Since C. brenneri subdivides an evolutionary branch between $C$. elegans and the siblings $C$. briggsae and $C$. remanei, comparisons of its genome with the others might help weed out nonfunctional DNA sequences that had failed to diverge in the sibling species. Comparisons with the more remote $C$. sp. 3 PS1010 might define more highly conserved sequences invariant within the Cae- norhabditis genus and not simply within the Elegans group. We therefore undertook a pilot project to sequence and analyze $\sim 0.5 \%$ of the genomes of C. brenneri and C. sp. 3 PS1010, including the Hox subcluster ceh-13/lin-39 (Streit et al. 2002; Stoyanov et al. 2003; Sternberg 2005; Wagmaister et al. 2006).

ceh-13 and lin-39 are a linked pair of Hox genes, orthologous to labial/HOXA1 and Sex combs reduced/HOXA5. Hox genes, an ancient class of developmental control genes, pose a special challenge to cis-regulatory analysis because they are not regulated as isolated loci. Instead, they are found throughout bilateria as conserved multigene clusters encoding paralogous transcription factors that are crucial for development, and that are expressed in complex spatiotemporal patterns requiring intricate transcriptional regulation (Garcia-Fernandez 2005; Lemons and McGinnis 2006). Hox genes not only function similarly in disparate animal phyla, but may also be regulated similarly (Malicki et al. 1992; Frasch et al. 1995; Popperl et al. 1995; Haerry and Gehring 1997; Streit et al. 2002; Garcia-Fernandez 2005), although few cis-regulatory elements shared by Hox clusters of different phyla have actually been found (Haerry and Gehring 1997; Streit et al. 2002).

Nematodes have only a single set of Hox genes. Several megabases of DNA and numerous non-Hox genes separate the $C$. elegans Hox cluster into three subclusters of two genes each: ceh13/lin-39, mab-5/egl-5, and nob-1/php-3 (Supplemental Fig. S1) (Aboobaker and Blaxter 2003). This differs from most vertebrate genomes, which have four or five versions of a single large, unfragmented Hox gene cluster (Lemons and McGinnis 2006). Some Hox genes have been lost in the C. elegans lineage, but all those present have vertebrate and arthropod orthologs (Clark et al. 1993; Maloof and Kenyon 1998; Aboobaker and Blaxter 2003; Stoyanov et al. 2003; Wagmaister et al. 2006). Cis-regulation is almost certainly confined within each $C$. elegans subcluster: The ceh-13/lin-39 subcluster is thus a natural experiment, in which two genes represent a cluster of vertebrate orthologs (Lemons and McGinnis 2006).

The ceh-13/lin-39 subcluster is vital for much anterior and mid-body development in C. elegans, but deciphering its cisregulation has been difficult and remains incomplete. It is large by C. elegans standards, with almost $20 \mathrm{~kb}$ of intergenic DNA encoding only a single microRNA gene. ceh-13 is required for both embryonic and postembryonic development; null ceh-13 mutations are lethal (Brunschwig et al. 1999). In the embryo, ceh-13 is expressed in the A, D, E, and MS lineages and is required for normal gastrulation (Wittmann et al. 1997). Two upstream regulatory sites have been reported to drive expression in the embryo, one of which also acts in the male tail (Streit et al. 2002; Stoyanov et al. 2003). Cis-regulation of post-embryonic ceh-13 expression, which includes the anterior dorsal hypodermis, anterior bodywall muscle, and ventral nerve cord (Brunschwig et al. 1999), is not yet well understood, especially in tissues where it is coexpressed with lin-39. While lin-39 is dispensable for viability, it is required for normal vulval development, migration of the QR and QL neuroblasts, muscle formation, and specification of VC neurons (Burglin and Ruvkun 1993; Clark et al. 1993; Wang et al. 1993; Clandinin et al. 1997; Grant et al. 2000; McKay et al. 2003). A recent study of the lin-39 promoter delimited several elements to $\sim 300$ bp by generating many transgenic reporter strains without using comparative genomics information; one of these elements was critical for vulval expression (Wagmaister et al. 2006). Our working hypothesis is that the complex expression of the ceh-13/lin-39 locus arises from the summed actions of independent conserved cis-regulatory elements.

\section{Genome Research}

www.genome.org 
We have dissected ceh-13/lin-39 cis-regulation through comparative genomics, and thus defined parameters likely to be useful for genome-wide analyses. This revealed several known and new regulatory elements, including one with functional similarity in mammalian Hox clusters.

\section{Results}

\section{DNA sequencing}

To enable comparisons to C. elegans, $1.1 \mathrm{Mb}$ of genomic sequences from C. brenneri and C. sp. 3 PS1010 were sequenced and assembled (Supplemental Tables S1, S2). This comprised $\sim 0.5 \%$ of each genome, assuming genome sizes roughly equal to C. elegans. The primary DNA sequence data were generally well assembled; the exception was a set of C. brenneri clones covering the mab-5/ egl-5 intergenic region, which may have suffered from high polymorphism found in gonochoristic Caenorhabditis species (Graustein et al. 2002).

\section{Sequence comparison}

We used MUSSA (multi-species sequence analysis; http://mussa. caltech.edu) to find preferentially conserved sequences. MUSSA is a $\mathrm{N}$-way sequence comparison algorithm, generalized from Family Relations (Brown et al. 2002), which integrates similarities among three or more genomes (see Methods). It compares, via sliding window, every frame in each participating sequence with every frame in all other sequences, allowing users to choose a window size and threshold of conservation for ungapped sequence matches (here called "MUSSA matches"). MUSSA produces an orientation-independent map of all one-to-one, one-to-many, and many-to-many transitive matches (Fig. 2). MUSSA matches highlight regions intolerant of insertions and deletions that may contain regulatory elements when found outside coding sequences (Cameron et al. 2005).

A number of parallel lines from visualizing MUSSA matches (at a given threshold of conservation) identified domains of similarity between the sequences, indicating the uniqueness and colinearity of potential regulatory elements (Fig. 2). Noise from repeats and low-complexity DNA sequence tended to create a cross-hatched pattern, reflecting many-to-many alignments that could be eliminated by raising similarity thresholds (Fig. 2A).

We initially performed two-way comparisons using a 30-bp window size, which minimized cross-hatched noise and had been useful in comparing mammalian genomes (T. De Buysscher, unpubl.). In principle, the threshold which gives $P \leq 0.05$ for spurious matches in a 30-bp window should be 19/30 identities in $1 \mathrm{~kb}$ of completely random sequence (Brown 2006). Since nonconserved sequence is not actually random, the real $P$-value must be larger. For thresholds of $\leq 21 / 30$, we found that cross-hatched connections marred the readout (Fig. 2B), while higher thresholds of $\geq 24 / 30$ revealed a much sparser set of nearly parallel connections (Supplemental Fig. S2A). As expected, comparisons of three or more genomic sequences allowed clean results at lower thresholds than pairwise comparisons, improving the signal-to-noise ratio (Fig. 2A,C; Supplemental Fig. S2A,B).

Three-way comparison of ceh-13/lin-39 sequences from $C$. elegans, C. briggsae, and C. brenneri with 30-bp windows identified several conserved regions (Fig. 2A). In C. elegans, the ceh-13/lin-39 locus includes $19 \mathrm{~kb}$ of intergenic sequence and $8 \mathrm{~kb}$ of intronic sequence, of which only $2 \%$ was highlighted in MUSSA matches at a threshold of $24 / 30(80 \%)$. This 50 -fold enrichment was the basis for experimental dissection of the locus. In contrast, comparison of C. elegans, C. briggsae, and C. sp. 3 PS1010 revealed substantially fewer MUSSA matches and gained no new alignments across the range of parameters (Fig. 2C; Supplemental Fig. S2C-F). After experimentally testing predicted elements, as reported below, we could re-evaluate the effects of window size and genome numbers, as well as determine the effects of using the C. remanei ceh-13/lin-39 locus (which was unavailable during the earlier part of our work).

Cis-regulatory elements operating during development are typically composed of multiple binding sites arrayed over several hundred base pairs (Davidson 2006; Li et al. 2007). We expected that not all of these binding sites would be preserved as ungapped sequence blocks. To ensure that our comparison parameters did not omit functional sequences from transgenic assays, we buffered each MUSSA match with 200 bp of flanking DNA on each side. Aligned features located close to each other were grouped into single regions for testing. In this manner, 11 different regions (N1-N11) were predicted to be functional (Fig. 3A). The intervening noncoding regions selected for study (I0-I9), being less conserved, were deemed less likely to be functional (Fig. 3A; Supplemental Table S3) but were also tested transgenically.

Four of the 11 conserved regions corresponded to sequences previously shown to have some function. Region N8 corresponds precisely to the microRNA mir-231 and its upstream promoter. mir-231 is expressed from embryonic through adult stages, but its biological role is unknown (Lim et al. 2003). Region N3 drives larval ventral nerve cord expression (pJW8) (Wagmaister et al. 2006); region N9 drives embryonic expression (enh450) (Streit et al. 2002); and a region including element N10 drives larval and male tail expression (271-bp enhancer) (Stoyanov et al. 2003). Because our comparison rediscovered elements of the ceh-13/lin39 subcluster previously shown to be important, it seemed likely that the newly defined blocks of similarity would also have biological activities.

\section{Expression in C. elegans}

We tested nine of the 11 strongly conserved regions, and all 10 intervening weakly conserved regions, for their ability to positively regulate expression; their repressor activity (if any) was not assayed. We did not retest the previously characterized N8 and $\mathrm{N} 10$, but did retest N3 and N9 to show that our assays reproduced published expression patterns in our reporter system (a $\Delta$ pes-10 basal promoter driving nuclear-localized GFP with an unc-54 $3^{\prime}$ untranslated region [UTR]). Background expression from the reporter is described in the Supplemental material, as are experiments showing that different basal promoters gave identical expression patterns in elements that were retested.

Most conserved regions drove expression in specific cell types (Table 1). In all cases, the described expression pattern was reproducible in multiple independent lines. Despite some spatial and temporal overlap, the expression patterns for each region were unique.

The intronic element N1 drove expression in vulval muscle, starting during the L4 larval stage and continuing through the adult (Fig. 4A). This element was well conserved with two MUSSA matches. Region N2 was expressed in the ventral nerve cord during the L1 larval stage (Fig. 4B). Expression of region N2 was also seen in some P cells and in the neural precursor Q cells, which are 
A

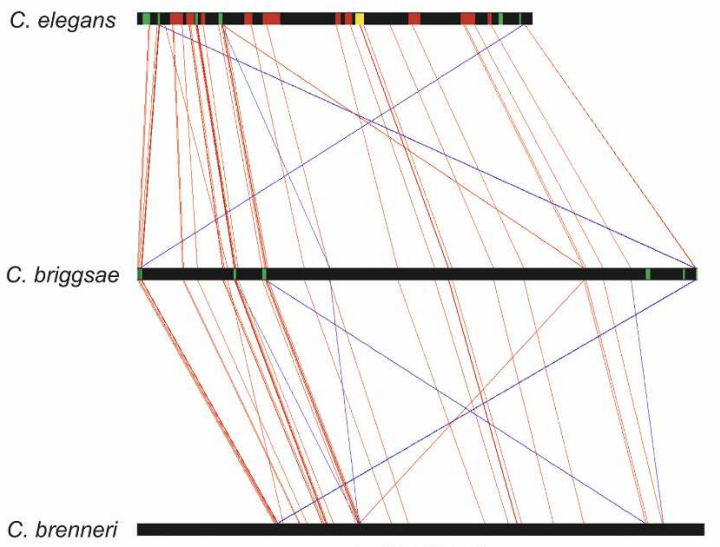

$24 / 30(80 \%)$

C

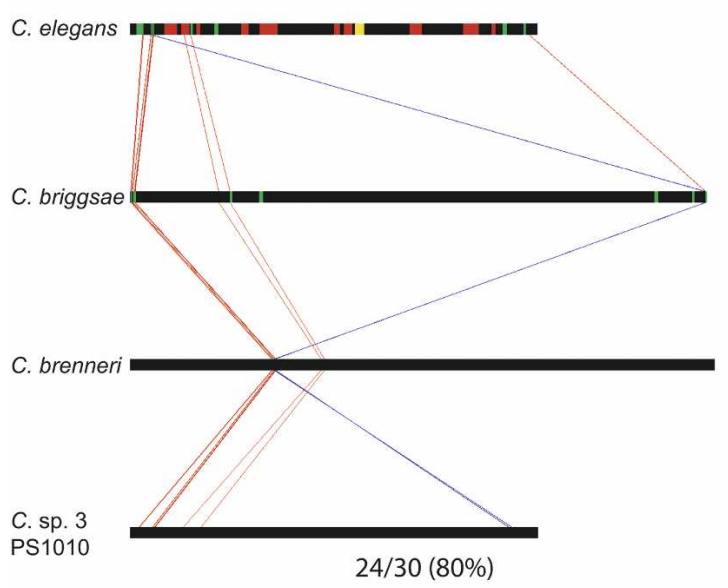

E

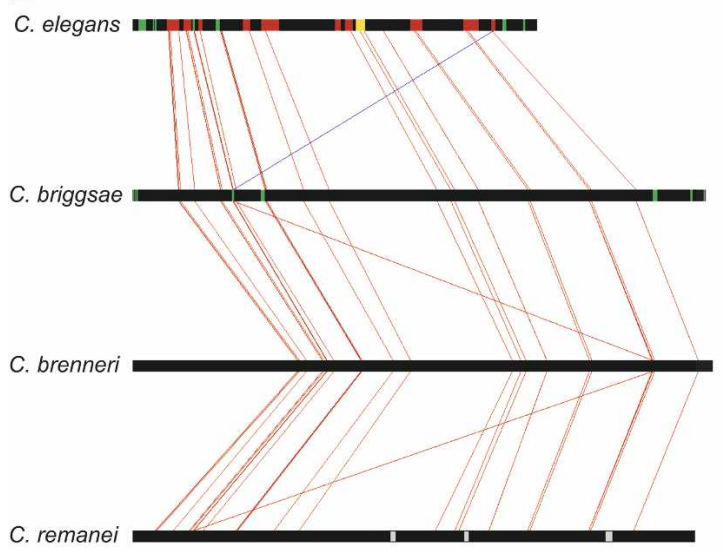

$18 / 20(90 \%)$
B

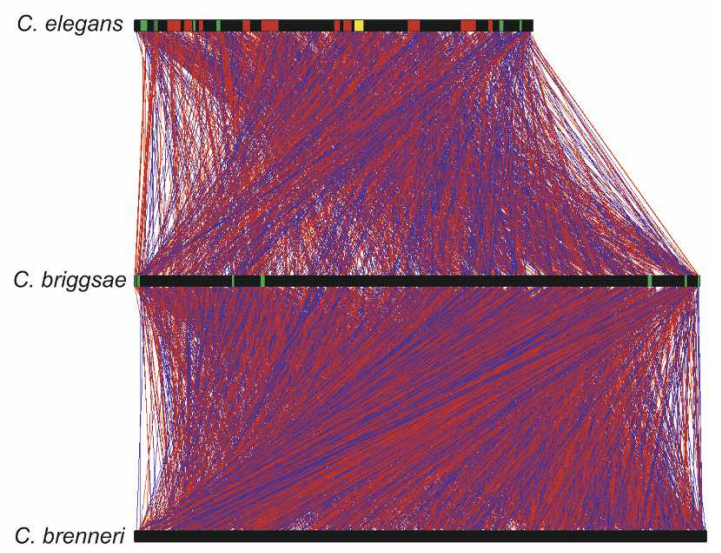

$21 / 30(70 \%)$

D

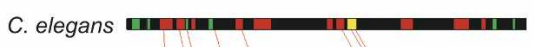

C. remanei

$27 / 30(90 \%)$

$\mathbf{F}$

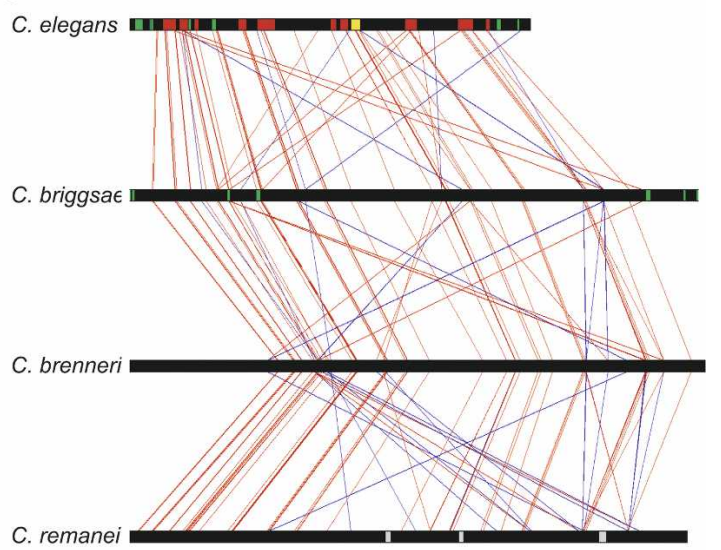

$14 / 15(93 \%)$

Figure 2. MUSSA comparisons highlighted ungapped sequence matches. Horizontal black bars represent the nematode sequences. The top sequence, C. elegans, has green sections for exons (with lin-39 on the left and ceh-13 on the right), red sections for each of the $\mathrm{N}$ regions, and a yellow section for region N8, which encompasses mir-231 and its promoter. The vertical lines highlight ungapped sequence MUSSA matches, with red lines for matches facing the same direction and blue lines for reverse-complement matches. The MUSSA matches represent transitive alignments, meaning they match across all sequences compared. (A) At high thresholds the vertical red lines are largely parallel, reflecting predominant colinearity of conserved sequence identified with $80 \%(24 / 30)$ sequence identity for a 30-bp window. As the threshold (identity/window length) decreases, more matches are identified by MUSSA but the noise also increases. (B) At a lower threshold, $70 \%(21 / 30)$, the graph is packed with many lines that cross each other, producing a cluttered, cross-hatched pattern. The number of species being compared may also be varied, giving a range of matches. Comparisons, using a 30-bp window, are shown between C. elegans, C. briggsae, and C. brenneri at $80 \%(24 / 30)(A)$ and C. elegans, C. briggsae, C. brenneri, and C. sp. 3 PS1010 at $80 \%(24 / 30)(C)$. The window size can also be varied at a constant threshold, as between $27 / 30(90 \%)(D), 18 / 20(90 \%)(E)$, and $14 / 15(93 \%)(F)$.

\section{Genome Research}

www.genome.org 
A

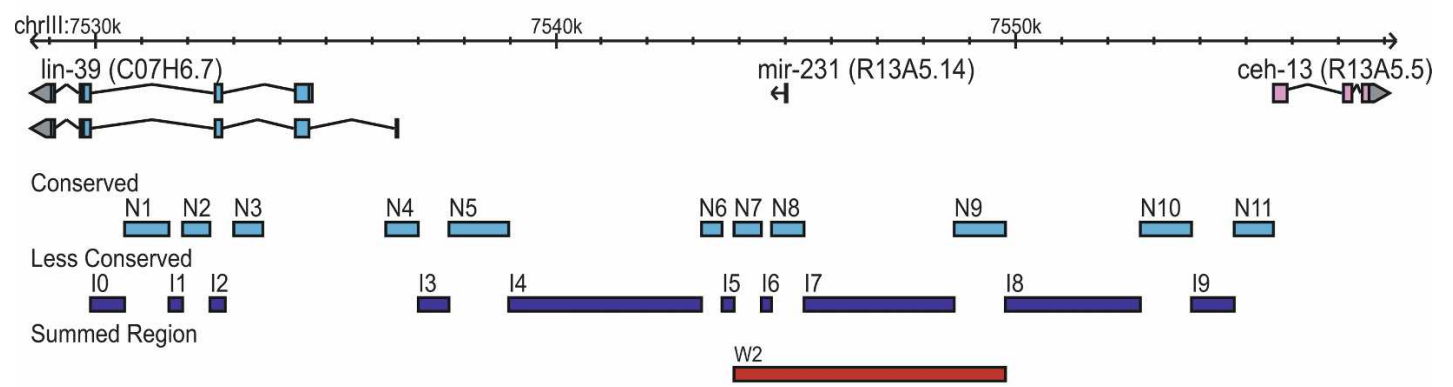

B

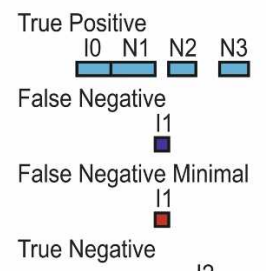

12

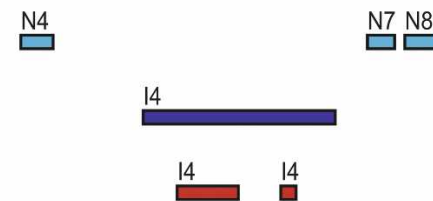

N7 N8

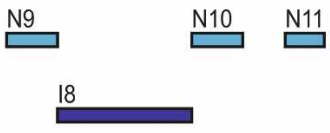

True Negative

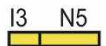

14

18

C

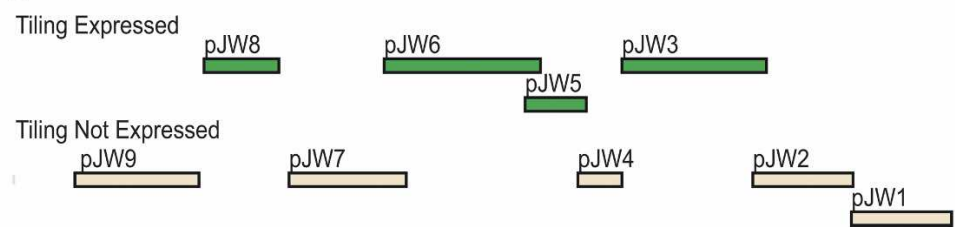

Figure 3. ceh-13/lin-39 Hox subcluster dissection based on sequence conservation. The ceh-13/lin-39 Hox locus was dissected into 21 sections for in vivo expression analysis based on the presence of MUSSA matches in a three-way alignment between $C$. elegans, $C$. briggsae, and $C$. brenneri. ( $A$ ) MUSSA matches were used to identify similar, presumably conserved regions ( $\mathrm{N}$ regions), which include the sequence match windows, 200 bp of $5^{\prime}$ and $3^{\prime}$ flanking sequence, and additional sequence for primer selection. The intervening, less-similar regions (I regions) located between the $\mathrm{N}$ regions were also tested. A "summed" region (W2) encompassing several component regions is shown as well. (B) With revised parameters of 100\% match of 15-bp windows, the regions were repartitioned and true positives, true negatives, and false negatives were identified. The minimal region to recover the observed expression in the false negatives is identified (Streit et al. 2002; Wagmaister et al. 2006). (C) The regions assayed in the tiling analysis from Wagmaister et al. (2006) are shown for comparison, noting which drove expression (green) and which did not (beige).

known to require lin-39 to regulate proper migration. N2 was also highly conserved: It consisted of two intronic MUSSA matches next to one another in all species except for C. sp. 3 PS1010, in which one match was inverted and moved $5^{\prime}$ with respect to lin-39. N2 occupies the same intron as N1, but is sufficiently separated (by 500 bp in C. elegans) to designate N1 and N2 as separate elements. Region N3, identified by one very wellconserved MUSSA match in the first intron of lin-39, was expressed in the hypodermal hyp7 cells in the late embryo and early L1 larvae (Fig. 4C) as well as in the V cells, P cells, and ventral nerve cord of the early L1 through L3 larvae. This expression pattern matched and expanded on that previously observed for this region (Wagmaister et al. 2006). Region N4 is in the proximal promoter region of lin-39; it drove expression in the ventral mid-body of the early embryo shortly after gastrulation (Fig. 4D). During early larval development N4 also drove expression in V6. Region N7 drove expression in the posterior bodywall muscle cells (Fig. 4E), starting in the late embryo and continuing through adulthood, and in the diagonal and longitudinal muscles of the male tail. Region N9 drove previously reported embryonic expression, along with previously unreported anterior bodywall muscle expression in L4 larvae and adults (Fig. 4F) (Streit et al. 2002). Region N11 was in the proximal promoter region of ceh-13 and drove expression in the anterior hypodermis of late embryos (Fig. 4G). Neither N5 nor N6 drove expression; this could be due to the limited conditions (e.g., non-dauer, noninfected, etc.) in which we scored the worms.

Potential regulatory sequences were found for both ceh-13 and lin-39. For conserved regions closer to ceh-13 (N9 and N11), observed patterns agreed well with expected ones (Wittmann et al. 1997; Brunschwig et al. 1999; Streit et al. 2002). Expression of lin-39 in the bodywall muscles, intestine, and central body region have all been described and were reproduced, for the most part, by conserved regions closer to lin-39: N1-N4 and N7 (Clark et al. 1993; Wang et al. 1993; Maloof and Kenyon 1998; McKay et al. 2003). Furthermore, expression in the anterior midbody is predicted for both transcription factors, meaning that regions N2-N4 could be acting on both genes. Published patterns for both ceh-13 and lin-39 may be incomplete, which would account for observed activities beyond those expected.

Each region drove a different expression pattern. The fusion of a large region (W2) that included both N7 and N9 drove expression in both anterior and posterior bodywall muscle, a simple summation of N7 (strictly posterior) and N9 (strictly anterior) expression patterns (Figs. $3 \mathrm{~A}, 4 \mathrm{H}$ ). It is unknown whether these regions regulate ceh-13, lin-39, mir-231, or all three genes.

We then asked what regulatory activities, if any, resided in the less-conserved regions between our conserved elements. 
Kuntz et al.

Table 1. Expression patterns of transgenic worms

\begin{tabular}{|c|c|c|c|}
\hline Region & Length & Stages & Expression pattern \\
\hline N1 & 964 & L4-adult & Vulval muscle \\
\hline N2 & 605 & $\begin{array}{l}\text { L1-adult } \\
\text { L1 }\end{array}$ & $\begin{array}{l}\text { Ventral nerve cord, Q cell daughters } \\
\text { P cells, Q cells }\end{array}$ \\
\hline N3 & 630 & $\begin{array}{l}\text { Embryo-L1 } \\
\text { L1-L3 }\end{array}$ & $\begin{array}{l}\text { Hyp7 } \\
\text { V cells, P cells, ventral nerve cord }\end{array}$ \\
\hline N4 & 697 & $\begin{array}{l}\text { Embryo } \\
\text { L1 }\end{array}$ & $\begin{array}{l}\text { Ventral midbody } \\
\text { V6 }\end{array}$ \\
\hline N5 & 1297 & Embryo-adult & Background (see below) \\
\hline N6 & 434 & Embryo-adult & Background (see below) \\
\hline N7 & 591 & Embryo-adult & Posterior bodywall muscle, nerve ring neurons, HSN \\
\hline N9 & 1120 & L4-adult & Anterior bodywall muscle \\
\hline N11 & 819 & Embryo & Anterior hypodermis \\
\hline 10 & 749 & $\begin{array}{l}\text { L2-adult } \\
\text { Embryo-L1 }\end{array}$ & $\begin{array}{l}\text { Coelomocytes, anterior ventral nerve cord } \\
V \text { cells, } \mathrm{P} \text { cells }\end{array}$ \\
\hline 11 & 289 & Embryo-adult & Seam cells \\
\hline 12 & 311 & Embryo-adult & Background (see below) \\
\hline 13 & 697 & Embryo-adult & Background (see below) \\
\hline 14 & 4182 & L3 & Sex myoblasts \\
\hline 15 & 280 & Embryo-adult & Background (see below) \\
\hline 16 & 216 & Embryo-adult & Background (see below) \\
\hline 17 & 3270 & Embryo-adult & Background (see below) \\
\hline 18 & 2906 & Embryo & Various \\
\hline 19 & 957 & Embryo-adult & Background (see below) \\
\hline W2 & 5892 & L4-adult & Bodywall muscle \\
\hline pPD107.94 & & L1-adult & $\begin{array}{l}\text { Background (anterior-most and posterior intestine, anterior-most bodywall muscle, } \\
\text { anal depressor cell, enteric muscle, excretory cell) }\end{array}$ \\
\hline pPD95.75 & & L1-adult & Background (see above) \\
\hline
\end{tabular}

The different regions of the Hox cluster that drove expression are listed with the corresponding temporal and spatial pattern. Regions with only "background" expression did not drive any unique detectable expression in our assays. Region N10 was previously described and not injected.

Four of the 10 less-conserved regions (I0, I1, I4, and I8) yielded expression apart from the expected background. Region I0 drove expression in the ventral posterior coelomocytes (Fig. 4I) and the two anterior inner longitudinal muscles of the male tail. This element had one MUSSA match that was strongly identified only when the window size was reduced to 15 or $20 \mathrm{bp}$. Region I1 drove expression in seam cells, starting with the embryo and continuing through to young adults (Fig. 4J). This element had no components strongly identified by MUSSA, with alignments appearing only at relatively low and noisy thresholds. Region I4 drove expression in the sex myoblasts through two cell divisions (Fig. 4K), as previously described by Wagmaister et al. (2006). Although expression was also reported in the Pn.p cells, we did not observe this, perhaps because I4 was not identical to the pJW5 region assayed by Wagmaister et al. (2006). I4 showed no MUSSA matches until a lower threshold of $22 / 30$ bp or a 20 -bp window was used, at which point the regions necessary for sex myoblast and ventral hypodermal Pn.p cell expression described by Wagmaister et al. (2006) were identified. Region I8 drove early embryonic expression, as previously reported (Streit et al. 2002). This region had a number of MUSSA matches that appeared as the threshold or window size was lowered.

\section{Testing for sequence necessity}

Our DNA regions from the ceh-13/lin-39 Hox subcluster contained not only blocks of ungapped sequence similarity, but also nonconserved sequences in which they were embedded. While these regions clearly drove expression in transgenic worms, our initial survey did not test whether the small conserved matches within them were crucial for regulatory activity. We therefore assayed in vivo constructs derived from some of the most highly conserved regions (N1, N2, N3, and N7; Supplemental Tables S3,
S4), in which we mutated the MUSSA match in C. elegans. For N7, mutating the MUSSA match completely eliminated expression in the posterior bodywall muscle, showing the match to be needed for regulation (Fig. 5). In contrast, the remaining mutated regions from N1-N3 had the same expression patterns as their respective wild-type constructs. The conserved matches in N1-N3 were themselves dispensable for regulatory activity, yet were closely associated with active regulatory sequences. Our data paralleled previous negative results of Wagmaister et al. (2006) for a point mutation in the N3 region (HP2), which was a possible Hox or Pbx binding site.

\section{Ultraconserved elements}

Hox clusters are evolutionarily ancient, sharing a common origin for all bilaterians (Garcia-Fernandez 2005; Lemons and McGinnis 2006), meaning that some cis-regulatory elements in C. elegans ceh-13/lin-39 might be conserved in other bilaterian phyla (Haerry and Gehring 1997; Streit et al. 2002). The following Hoxclusters were searched for any possible MUSSA matches to our conserved elements: the single Hox clusters of Drosophila melanogaster, Aedes aegypti (mosquito), Anopheles gambiae (mosquito), Apis mellifera (honey bee), Branchiostoma floridae (lancelet), Capitella sp. I (polychaete worm), Helobdella robusta (leech), Lottia gigantea (snail), Schistosoma mansoni (trematode), Schmidtea mediterranea (flatworm), and Tribolium castaneum (beetle); the four Hox clusters of mouse and human; and the seven Hox clusters of zebrafish. In each of these genomes we found several matches of uncertain significance. We therefore searched orthologous Hox regions for recurrent patterns of MUSSA matches (Fig. 6A). In newly characterized phyla, for which several related genomes had not yet been sequenced, this approach did not help to evalu-

\section{Genome Research}

www.genome.org 


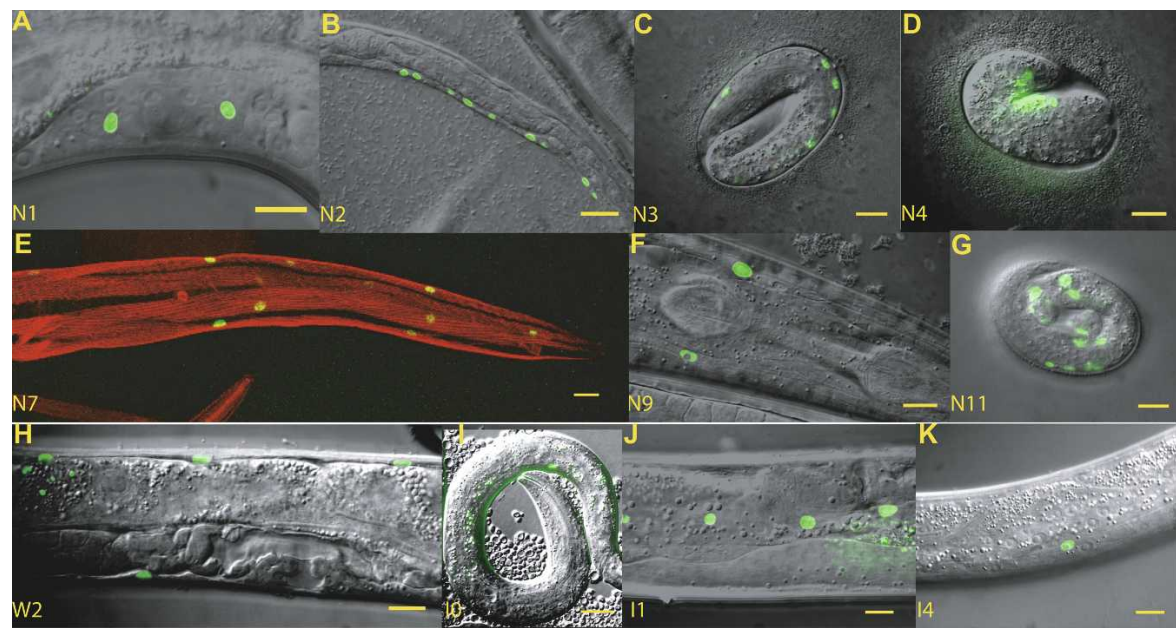

Figure 4. In vivo expression patterns. Many well-conserved and some poorly conserved regions drive independent and reproducible expression. Expression is observed in a variety of tissues that largely agree with published antibody staining for ceh-13 and lin-39. $(A)$ Element N1 directs expression in the L4 to adult vulval muscles. $(B)$ Element N2 directs expression in the late embryo through L2 in the ventral nerve cord and P cells. (C) Element N3 directs expression in late embryonic through L3 hyp7, and in the V cells and $\mathrm{P}$ cells soon after hatching. (D) N4 directs expression in cells of the AB lineage in the dorsal mid-body during the comma stage. $(E)$ N7 directs expression in the posterior bodywall muscle in the late embryo through the adult. N8 contains mir-231 and was not assayed. ( $F$ ) N9 directs expression in the anterior bodywall muscle in the adult. (G) N11 directs expression in anterior late embryos. (H) W2, a large region spanning N7, N8, and N9, directs expression in both the anterior and posterior bodywall muscles, demonstrating additive coexpression of N7 and N9. (I) I0 directs expression in the posterior ventral coelomocyte. (D) 11 directs expression in the seam cells. $(K)$ I4 directs expression in the SM cells. All scale bars are equal to 10 microns. For background expression from the reporter, see Supplemental material and Supplemental Figure S4.

ate hits; but it was useful in vertebrates and insects, for which many related genomes were available.

In both mouse and human, N3 and N7-like MUSSA matches were paired with each other in the HOXA cluster near the ceh-13 and lin-39 orthologs, HOXA1 and HOXA5, respectively. Scans of the HOXA clusters in dog, opossum, platypus, and frog also revealed this pairing (Fig. 6A). Among the vertebrates alone, sequence conservation was high, indicating that these hits were located in functionally important DNA (Fig. 6B), although these sites had not been previously described. Using a low threshold, the matches showed similarity through nematodes and vertebrates, with the N3-like MUSSA match just 3' of HOXA1 being more similar (86\%) than the N7-like MUSSA match just $5^{\prime}$ of HOXA5 (73\%) (Fig. 6C; Supplemental S3A). Similar searches within 11 Drosophila species yielded matches highly conserved among insects, but with only low levels of similarity to either nematodes or vertebrates.

To test whether the interphylum similarities revealed functional sequences, we cloned a 700-bp region of mouse Hox genomic DNA centered on the mouse N3-like MUSSA match and a 650-bp region centered on the N7-like MUSSA match, each containing local sequence conserved among mammals. We assayed both regions in C. elegans transgenes. The mouse N3-like region drove almost the same expression pattern as the C. elegans N3 region (Fig. 6D) in hyp7, P cells, $\mathrm{V}$ cells, and the ventral nerve cord, with discordant activity in only a few extra anterior hypodermal cells. Whereas C. elegans N3 was previously predicted to include a $\mathrm{Hox} / \mathrm{Pbx}$ autoregulatory site for lin-39 (Wagmaister et al. 2006), the mouse N3-like MUSSA match is found closer to Hoxa1 (a ceh-13 ortholog) than to Hoxa4 (a lin-39 ortholog). N3 could be a general Hox binding site, or its role may have changed over time. In contrast, the mouse N7like region failed to drive the posterior bodywall muscle expression as the C. elegans N7 region did, though its background expression level was noticeably increased (Supplemental Fig. S4A).

If N3's similarities between nematodes and vertebrates result from common descent, N3-like matches should exist in other animal phyla. We found co-occurrence of two top-scoring MEME motifs and a MUSSA match in the nematodes, vertebrates, $B$. floridae, Capitella sp. I, H. robusta, and $S$. mansoni (Supplemental Fig. S3B; Supplemental material). MUSSA comparison of N3-like sequences in nematodes, vertebrates, and $B$. floridae yielded a $70 \%$ match, while a comparison of nematodes, vertebrates, $S$. mansoni, and $H$. robusta yielded a $65 \%$ match (Supplemental Fig. S3B). These matches encompass deuterostomes, ecdysozoa, and lophotrochozoa-all of the major divisions of bilateria. Thus, we interpret the N3 site to be evolutionarily conserved rather than convergent.

\section{Threshold revision}

Having had some success with our initial parameters for ungapped sequence comparison, we then adjusted them empirically and retested them computationally against well-characterized genes in the hope of optimizing our parameters for genome-wide analysis. Initially, nine of the 11 regions ( $82 \%$ ) identified by conservation gave expression, while three of the 10 less conserved regions (30\%) gave expression; this was promising, but left room for possible improvement. When we tried lower thresholds or smaller windows, MUSSA found matches in some regions that had previously given no hits despite having regulatory activity (and that we had originally classified as false negatives). We therefore optimized the parameter settings and genome combination to achieve the best yield of functional elements while keeping false positives to a minimum (Fig. 2D-F; Supplemental Figs. S2G-L, S5, and S6). A 15-bp window and perfect conservation between $C$. elegans, C. briggsae, C. remanei, and C. brenneri identified MUSSA matches in $77 \%$ of all expressing regions with no false positives (Fig. 7A). Using a different window size (14 or 16-30 bp) decreased the resolution and efficiency (see Supplemental material; Supplemental Figs. S5, S6A,B). Including C. sp. 3 PS1010 sequences adequately selected the top hits, but only at the expense of eliminating many other hits and considerably reducing predictive power (Fig. 7B). Though the four Elegans group species together gave the best analysis, inclusion of $C$. remanei masked matches in the I4 region (Supplemental Fig. S2E; see Discussion).

The intervening regions were often much larger than any conserved region. For instance, region I4 was $4.2 \mathrm{~kb}$; however, the subsection of I4 sufficient to drive expression was $1.6 \mathrm{~kb}(38 \%$ of I4) (Wagmaister et al. 2006). Likewise, region I8 was $2.9 \mathrm{~kb}$, but expression could be recapitulated with only $0.7 \mathrm{~kb}$ within it $(24 \%$ of I8) (Streit et al. 2002). Thus, the density of regulatory regions 


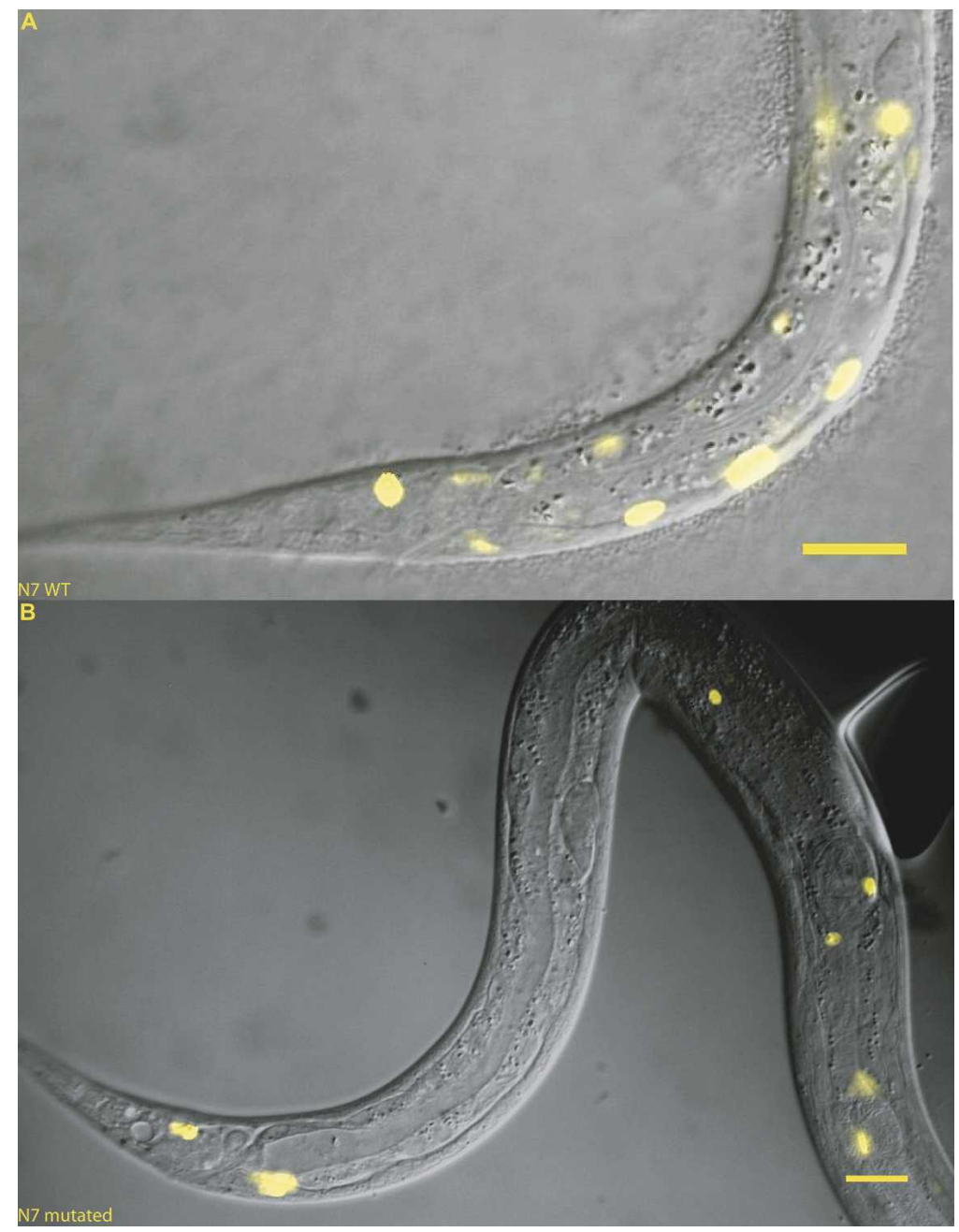

Figure 5. Mutating a conserved window in N7 knocked out expression. Element N7 (592 bp) normally drives expression in the posterior bodywall muscle $(A)$. (B) When the 20-bp MUSSA match was reversed, all expression in the posterior bodywall muscle was abolished. Scale bars, 10 microns.

\section{Discussion}

This study found four known and seven new cis-regulatory elements in the ceh13/lin-39 Hox subcluster of C. elegans, using ungapped sequence conservation across four genomes and verification by transgenic analyses. Remarkably, one conserved element's mouse counterpart recapitulated the native nematode expression pattern. The observed expression patterns generally paralleled those found by prior antibody staining and expression from the parental undissected promoters, suggesting that the union of these cis-regulatory elements drives the entire endogenous expression pattern, and that we have identified most cisregulatory regions of $c$ h-13/lin-39 (Clark et al. 1993; Wang et al. 1993; Wittmann et al. 1997; Maloof and Kenyon 1998; Brunschwig et al. 1999; Streit et al. 2002; McKay et al. 2003).

For ceh-13/lin-39, our first parameters for sequence conservation worked well, even though we later improved them empirically. They identified 11 possible elements, of which nine showed function experimentally, leaving two false positives-a threefold enrichment for functional regulatory elements compared with simple, unselected tiling. With revised parameters, $100 \%$ of the computationally identified elements were functional. For these nematode sequences, we found that MUSSA predicted function with highest reliability and resolution when we used windows of $15 \mathrm{bp}$. Smaller windows gave noisier alignments with poor resolution, while larger windows tended to

within nonconserved sequences is probably even lower than our data indicate (Fig. 3B). When compared with tiling, as performed by Wagmaister et al. (2006), conservation-based analysis confers an efficiency advantage, with $100 \%$ instead of $40 \%$ specificity (Fig. 3C; Wagmaister et al. 2006).

To test whether the revised parameters are useful outside the Hox cluster, we analyzed the previously described C. elegans genes hlh-1, myo-2, myo-3, and unc-54 (Okkema et al. 1993; Krause et al. 1994). These were chosen for analysis because their promoter dissections had been screened for expression across all tissues, unlike most studies that identify positive expression in a specific tissue but did not screen for negative activity across other tissues. Using our strict 15-bp threshold and technique of including 200 bp of flanking DNA, all known regulatory elements of the myosin genes myo-2, myo-3, and unc-54 (Okkema et al. 1993) were identified with no false positives (Supplemental Fig. S7). For the hlh-1 locus, two of four regulatory sites (Krause et al. 1994) were recovered at a lower threshold. Therefore, MUSSA predictions were accurate at some non-Hox loci, but as in the Hox locus itself, some functional elements could not be identified this way. miss shorter conserved sequences with regulatory activities. These parameters correctly rediscovered regulatory regions in other well-characterized genes, but made some errors, suggesting additional possible refinements as functional data becomes available at other loci. However, we do not expect that this method, used on its own, will discover all elements. We also expect parameters to change when the set of compared genomes is changed, as we have already found. For instance, the conserved regions for vertebrate Hox sequences (e.g., the N3-like mouse region) were much longer than in nematodes, and could be detected at a lower MUSSA threshold with a larger window size. Such differences in sequence conservation might arise from different rates and types of mutations, or from altered selection pressures.

Our aim was to efficiently predict new elements with bona fide biological activity, accepting that this runs the risk of missing some regulatory regions. Nevertheless, correctly identifying even two-thirds of all C. elegans regulatory elements with a low false-positive rate, as we did prior to refinement, could significantly advance our knowledge of the worm regulatory genome. Recent uses of sequence constraint in vertebrates have

\section{Genome Research}

www.genome.org 
A Human

Mouse ${ }_{\mathrm{N} 2-1 \mid}$

Opossum

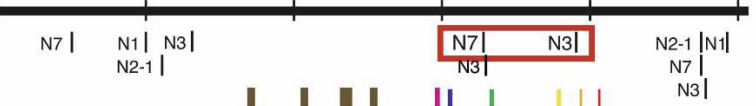

Platypus

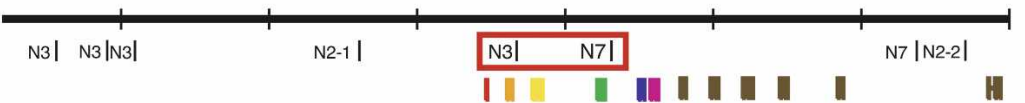

Frog

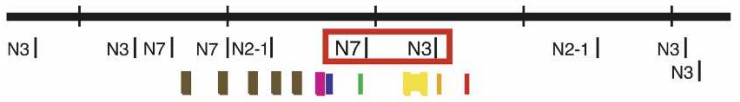

Zebrafish

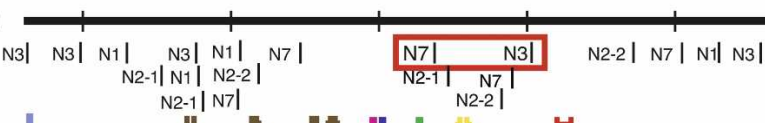

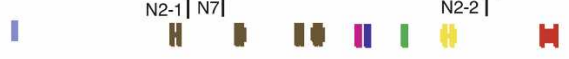

$\mathbf{B}$
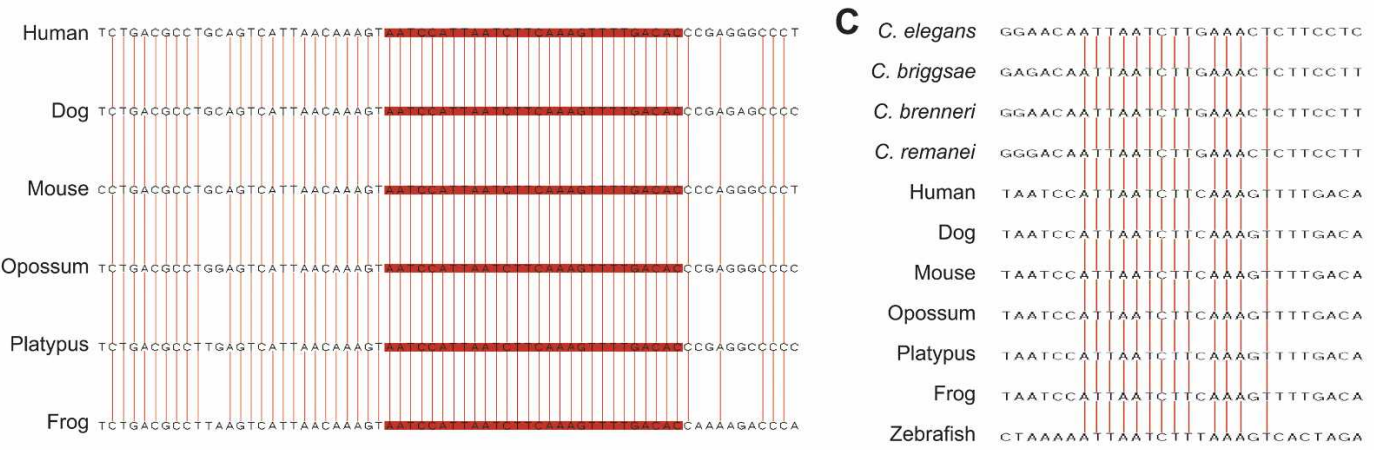

D

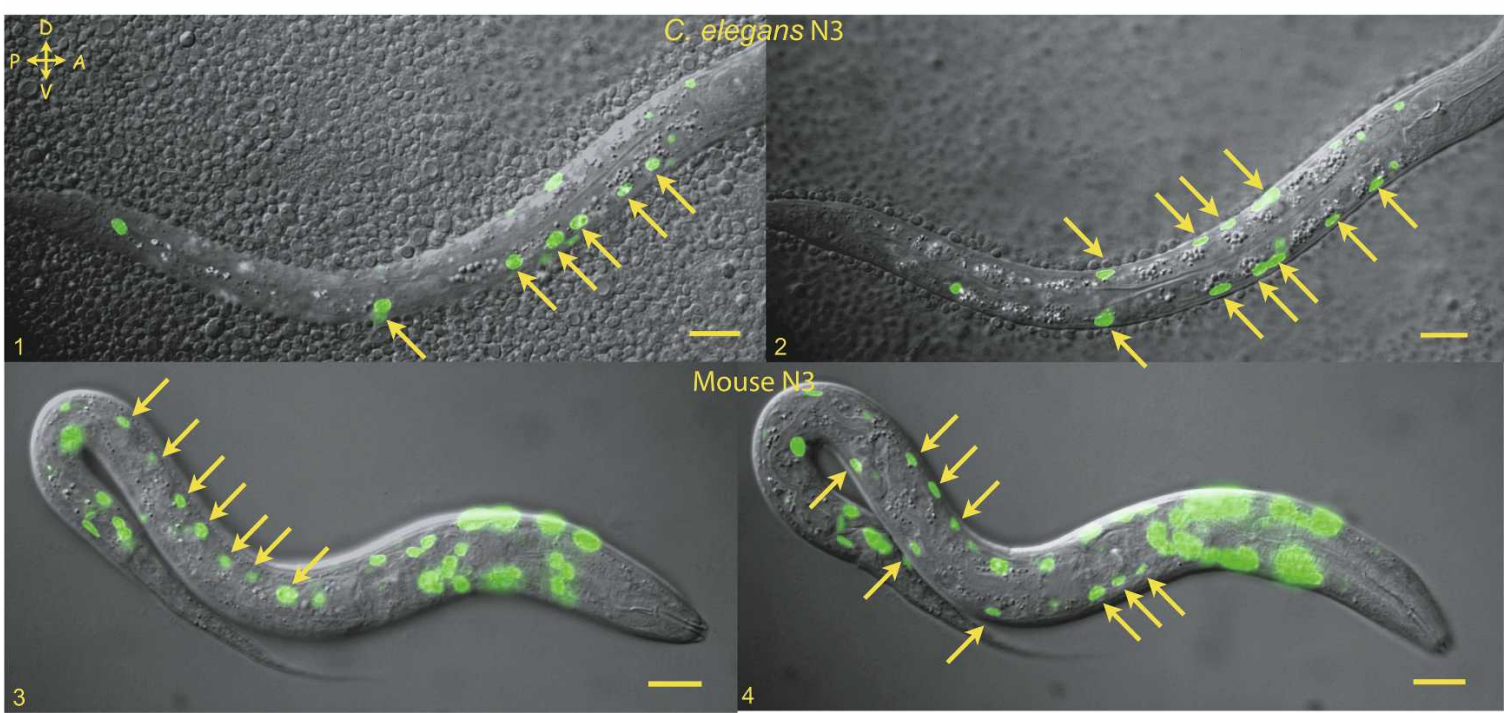

Figure 6. N3 cis-regulatory elements from either nematodes or vertebrates drove expression equivalently. $(A)$ MUSSA analysis was used to identify any ungapped matches between nematodes and various vertebrates. Synteny of two elements, N3 and N7 highlighted by red boxes, suggested the match was not noise. All figures are to the same scale (hash marks represent 50-kb distances), with the regions examined in each case bounded by the next 5' or 3' curated genes on the chromosome. The Hox genes are color coded: (red) HOXA1, (orange) HOXA2, (yellow) HOXA3, (green) HOXA4, (blue) HOXA5, (purple) HOXA6. (B) Apparent conservation of N3 among vertebrates was very high, with similarity still at $100 \%$ in a $30-\mathrm{bp}$ window. Vertical red lines represent base conservation between all six species. (C) N3 sequences shared $75 \%$ identity, using a 20-bp window, across 11 vertebrate and nematode species. $(D)$ A mouse N3-like region drove expression in C. elegans that was almost identical to that driven by the $C$. elegans $\mathrm{N} 3$ region. Expression is seen in L1 larvae in the V cells on the left (D1, D3), and P cells and hypodermal syncytium on the right (D2, D4). Additional expression in observed in the head with the mouse construct. Scale bars, 10 microns. 
A

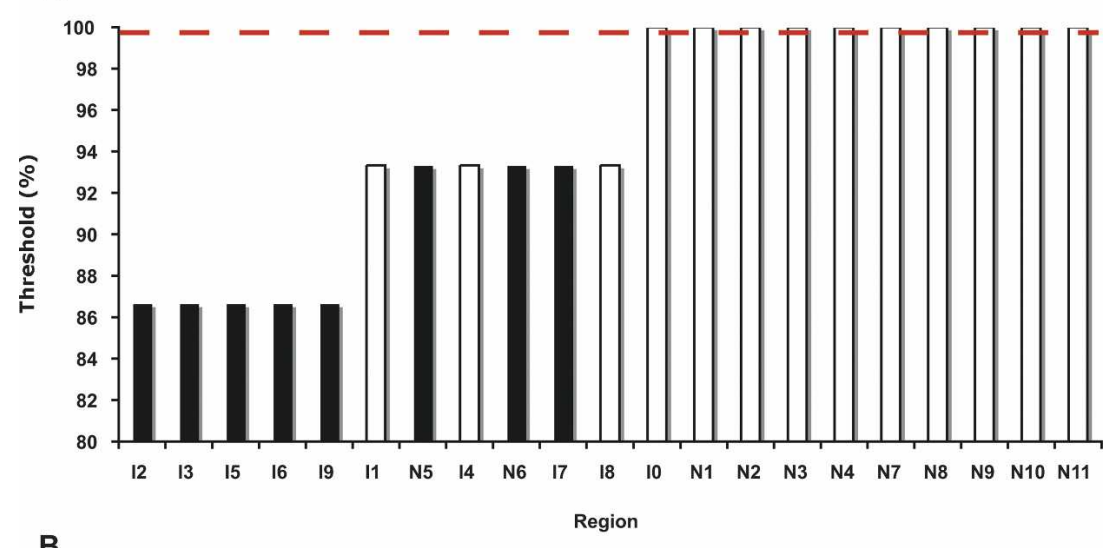

B

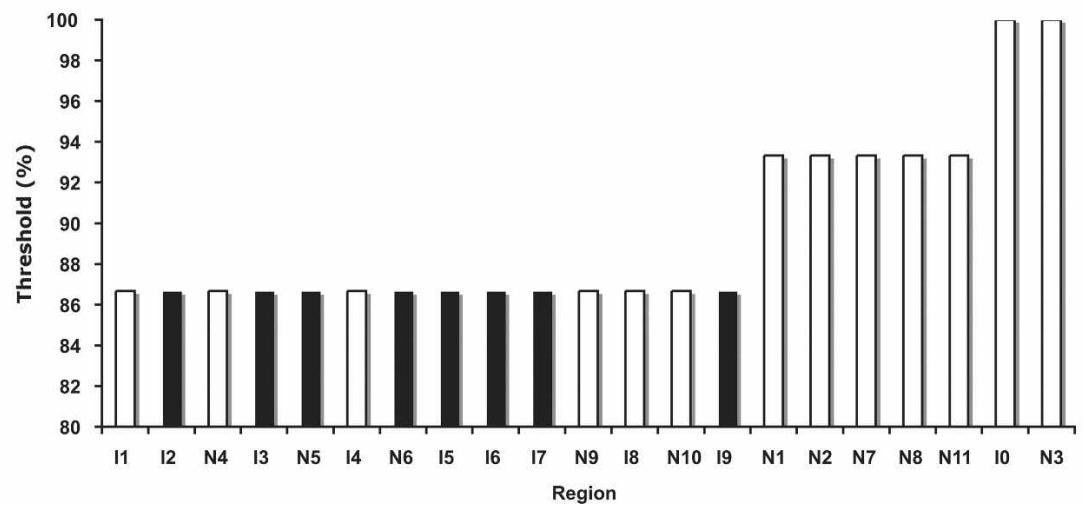

Figure 7. Revising MUSSA parameters for well-conserved regions. (A) A 15-bp window and four-way comparison among C. elegans, C. briggsae, C. brenneri, and C. remanei identified the thresholds at which MUSSA matches are observed within a region. Regions capable of driving expression are shown in white and those not capable of driving expression are shown in black. With a threshold of $100 \%$, there is a $77 \%$ recovery of expressing regions with perfect specificity. (B) Using five-way comparisons and a 15-bp window among the four above species and C. sp. 3 PS1010, the thresholds where conservation was still observed were identified for each element. The predictive power for identifying functional regions is considerably reduced from the four-way comparison.

been less sensitive in finding regulatory elements, perhaps because vertebrates undergo qualitatively different regulation (Pennacchio et al. 2006; McGaughey et al. 2008), although there are many differences, both biological and methodological, between their studies and this one. Only a representative subset of regulatory sites are needed to derive refined, genome-wide motifs in C. elegans, as we did with N2-1 (Supplemental material), which can then be statistically correlated with traits of their neighboring genes (Wenick and Hobert 2004; Mortazavi et al. 2006; Etchberger et al. 2007).

If a given regulatory element is mutated or fragmented in some species, comparing it with different sets of related species can still allow detection of that element. Such regulatory mutations are known to be responsible for subtle evolutionary changes in the salt resistance and excretory canal phenotypes of C. elegans, which have diverged from the ancestral phenotypes retained in C. briggsae and C. brenneri (Wang and Chamberlin 2004). The most striking difference in conservation we observed was between Elegans group species and the outlying $C$. sp. 3 PS1010. Four-way comparison of C. elegans, C. briggsae, C. brenneri, and $C$. remanei predicted the most regulatory elements, many of which could only be detected in C. sp. 3 PS1010 with much lower and noisier thresholds. Although all regions identified with C. sp. 3 PS1010 drove expression, there was no added benefit from this comparison; rather, it increased the false-negative rate. Similarly, neither lin-3 nor lin-11 in C. sp. 3 PS1010 had the organization or the sequence motifs of the genes in the Elegans group species (Supplemental Fig. S8; Supplemental Table S5). Additional Caenorhabditis genomic sequences should clarify which parts of the C. elegans genome encode speciesor group-specific traits.

The regulatory organization of the ceh-13/lin-39 locus appears to be modular, with each regulatory element functioning independently in transgenes: The expression output of two elements on a single DNA fragment (N7 and N9 on W2) or of four coinjected elements (N1, N2, N3, and N7) matched the sum of their individual activities. Nevertheless, the linear order of conserved elements across the ceh-13/lin-39 locus has been conserved between the different Caenorhabditis species, including the relatively distant C. sp. 3 PS1010, suggesting that element order is under selective pressure. Among the elements, there is also potential for some functional redundancy, as has been noted in mammals (e.g., Ahituv et al. 2007). ceh-13, for example, is expressed in the larval ventral nerve cord (Brunschwig et al. 1999) and three different elements drive expression there.

Multiple regulatory elements distributed throughout large introns and flanking sequences control many metazoan genes expressed in complex spatiotemporal patterns (Woolfe et al. 2005; Davidson 2006; Pennacchio et al. 2006) and ceh-13/lin-39 follows this trend. Only two of the nine expressing regions were located within the proximal 2 -kb promoter sequences of ceh-13 or lin-39, and four were in lin-39 introns. We did not assay for the effect that these regions had on ceh-13, lin-39, or mir-231 expression. Other examples of distal elements in C. elegans include remote regulation of ceh-10 and osm-9 (Colbert et al. 1997; Wenick and Hobert 2004).

Conservation analysis helped define elements without inadvertently splitting them, a hazard in blind deletion analysis. Moreover, it may have freed elements from inhibitory sequences, as we found that some large segments were less active when assayed than their subdomains. The entire second intron of lin39 yielded no expression in a prior study (Wagmaister et al. 2006), but we identified four different active cis-regulatory elements (N1, N2, I0, and I1) by subdividing the region. One possibility is that poorly conserved DNA separating ceh-13/lin-39 elements harbors hidden regulatory functions that our assay misses, such as repression. The basal promoter construct we used to screen for in vivo enhancer activity is not expected to detect isolated transcriptional silencers or insulators. This could explain moderately conserved but inactive regions, as might enhancers 
dependent on untested culture conditions or promoter-specific interactions with regulatory elements (Wenick and Hobert 2004; Etchberger et al. 2007).

Although large regions can be split into smaller functional components (such as the W2 region dividing into N7, N8, and $\mathrm{N} 9$, and the lin-39 intron dividing into N1, N2, I0, and I1), further dissection of functional elements might simply disrupt them, yielding weak and variable expression. This has been observed for ceh-13 male tail expression when multiple sites within N10 were mutated (V. Wegewitz and A. Streit, pers. comm.).

Biologically relevant sequence motifs often appear in or near the best-conserved regions, even if the MUSSA matches themselves are not essential for regulatory activity. For instance, two conserved MUSSA matches <200-bp apart identify the element N9; but a known motif that is not part of either conserved window is located next to them, and is necessary for proper regulatory function (Supplemental Fig. S9A). In four of five mutageneses, changing just one conserved feature had little effect, which is consistent with functional redundancy often seen in multi-site regulatory elements. Our assays used injected transgenes, for which multiple copies generally exist of a cloned reporter (Mello and Fire 1995); this might have provided a relaxed context for gene expression, tolerating the loss of "redundant" sites actually required in vivo. A site that subtly controls the quantity or spatiotemporal pattern of gene activity could easily lack an observable impact on GFP expression. Thus, it is important to test not only conserved sequences for regulatory activity, but the sequences near them.

The apparent conservation of N3 and N7 regions across phyla suggests that they predate the divergence of bilateria. Although mouse N7 was not active in the cross-phylum assay, the mouse N3-like region was strikingly positive and contains a potentially autoregulatory Hox/Pbx binding site. To test regulatory elements for functional conservation between different animal phyla, Drosophila enhancers and promoters have been compared with those of C. elegans and mammals: This generally involved isolating an enhancer or promoter with a known expression pattern in a donor organism, and testing it transgenically for similar expression in a second, distantly related organism (Malicki et al. 1992; Frasch et al. 1995; Popperl et al. 1995; Haerry and Gehring 1997; Streit et al. 2002; Ruvinsky and Ruvkun 2003). With nematode and mouse N3 regions, we instead tested the donor enhancer for activity equivalent to that already defined for its ortholog in the recipient species. This provides an alternative for comparisons over very long evolutionary distances, across which anatomical similarities may not be obvious. Moreover, additional MEME motifs, one of which may have been independently identified in mammals (as LM115 and LM171 of Xie et al. [2007]) (Supplemental Results), are shared by the vertebrate and nematode sequences. Based on these in vivo data and computational analyses, we consider N3 a pan-phyletic regulatory sequence. Such sequences may be rare, and only present in the most ancient regulatory loci, such as the ParaHox or NK clusters (Garcia-Fernandez 2005).

\section{Methods}

\section{General methods and strains}

We obtained Caenorhabditis elegans, C. brenneri CB5161, and C. sp. 3 PS1010 from the CGC strain collection and cultured them on OP50 at $20^{\circ} \mathrm{C}$, using methods standard for C. elegans (Sulston and Hodgkin 1988). unc-119(ed4) hermaphrodites were microinjected with a mixture of $60 \mathrm{ng} / \mu \mathrm{L}$ unc-119 vector, $12 \mathrm{ng} / \mu \mathrm{L}$ unpurified fusion product, and either $100 \mathrm{ng} / \mu \mathrm{L}$ pBluescript or $100 \mathrm{ng} / \mu \mathrm{L}$ digested genomic DNA to generate transgenic animals (Mello and Fire 1995; Kelly et al. 1997). All noted expression patterns were observed in two or more independent transgenic lines. In nonexpressing lines, at least 16 hermaphrodites from three independent lines (each line driving background GFP to guarantee GFP's functionality) were observed at each stage (early embryos, late embryos, L1-L4 larvae, young adults, and mature adults) with $100 \times$ magnification; males and dauers were observed for some, but not all, reporter lines.

\section{DNA preparation}

DNA was prepared by standard methods (Sulston and Hodgkin 1988). pEpiFos-5 (Epicentre), based on pBeloBAC11 (Birren et al. 1999), was used as the fosmid library vector. Fosmid sequences were shotgun sequenced and assembled into contigs by the Department of Energy's Joint Genome Institute at Walnut Creek (http://www.jgi.doe.gov/sequencing/protocols).

\section{Sequence analysis}

Sequence contigs from JGI were initially linked by BLASTN (Korf et al. 2003) and then merged with the revseq and megamerger functions of EMBOSS (Olson 2002). Our C. brenneri data had 22 genomic contigs, totaling 680,633 nucleotides (Supplemental Table S1). Our C. sp. 3 PS1010 data had seven genomic contigs, totaling 417,129 nucleotides (Supplemental Table S2). Gene predictions were made with Twinscan 3.5 running in single-species mode with C. elegans parameters (Wei et al. 2005); predicted protein sequences were extracted with BioPerl (Stajich et al. 2002). C. brenneri and C. sp 3 PS1010 protein sequences were tested for orthology against one another and against the proteincoding gene sets of $C$. elegans, C. briggsae, and C. remanei (from the WS170 release of WormBase) with OrthoMCL 1.3 (Li et al. 2003). Inferred ortholog groups were considered specific (i.e., unique) if they contained only one C. elegans gene, and only one gene from either $C$. briggsae or C. remanei. Our C. brenneri contigs encode 141 predicted proteins of $\geq 100$ residues in length, of which 88 have unique C. elegans orthologs (Supplemental Table S1). Our C. sp. 3 PS1010 contigs encode 86 predicted $\geq 100$ residue proteins, 68 with C. elegans orthologs (Supplemental Table S2). SVG genomic sequence images were generated by GBrowse for nematodes and vertebrates at the Wormbase (http:// www.wormbase.org) and UCSC Genome Browser (http:// genome.ucsc.edu) websites.

MUSSA (mulitple species sequence analysis) (http:// mussa.caltech.edu), a program written in $\mathrm{C}++$ with a Python controlled user interface, was used to identify evolutionarily conserved sequences. MUSSA uses N-way transitivity (all-against-all) so that only windows passing the selected similarity threshold across all species are reported as alignments. No sequences were repeat-masked in the comparisons performed here, though use of MUSSA in other phyla may benefit from masking as a preprocessing step (T. De Buysscher, D. Trout, and B.J. Wold, unpubl.).

For regulatory element dissection in the ceh-13/lin-39 cluster, published sequences from C. elegans, C. briggsae, and C. remanei (http://www.wormbase.org) were used with novel sequences from C. brenneri and C. sp. 3 PS1010. The mab-5/egl-5 Hox cluster comparisons used sequences from C. elegans, C. briggsae, and C. remanei. Additional comparisons with non-nematodes used sequences from all of each organism's available Hox clusters (http://www.ensembl.org; http://genome.ucsc.edu; http:// www.genedb.org/genedb/smansoni; http://racerx00.tamu.edu; and http://genome.jgi-psf.org). Known regulatory regions of 
non-Hox genes were linked from C. elegans to other species using MUSSA.

\section{MEME}

Multiple EM for Motif Elicitation (MEME) v3.5.4 was used to identify nonaligned motifs shared by different animal phyla (http://meme.sdsc.edu/meme) (Bailey and Elkan 1994). MEME motifs from the N3 element were tested for similarities to previously published genomic motifs by examining two 14-nt human sequences with up to two mismatches against JASPAR CNE (Bryne et al. 2007; Xie et al. 2007).

\section{Transgene design and construction}

PCR fusions were generated using standard protocols, essentially as in Hobert (2002). Genomic DNA and the cosmids R13A5 and C07H6 (from A. Fraser and R. Shownkeen at the Sanger Institute) were used as sequence templates. The Fire Lab Vector pPD107.94 was used as the template for the $\Delta p e s-10:: 4 \mathrm{X}$ NLS:: eGFP::LacZ::unc-54 sequence (Mello and Fire 1995). The Fire Lab Vector pPD95.75 was used as the template for the "promoterless" eGFP:: unc-54 sequence (Etchberger and Hobert 2008), used as a control in four constructs to demonstrate identical expression patterns under different basal promoters. Mutation primers were used to mutate target sites in plasmids. The mutated and sequenced enhancers were fused to Fire Lab Vector pPD122.53, where GFP was replaced with YFP, to give a $\Delta$ pes$10:: 4 X-N L S::$ YFP :: unc-54. GFP was replaced with CFP for unmutated controls. We mutated conserved sequences by reversal, not reverse complementation; such reversal maintained the base content, but was expected to destroy any sequence-specific binding of transcription factors. Complete methods are described in the Supplemental material.

\section{Acknowledgments}

We dedicate this study to the memory of E.B. Lewis, who pioneered the analysis of Hox clusters at Caltech. We thank C.T. Brown for discussions, N. Mullaney for work on an early version of MUSSA, E. Moon for aid in fosmid library construction, and E. Rubin and his colleagues at the DOE JGI for fosmid sequencing and assembly. We thank L.R. Baugh, C.T. Brown, C. Dalal, J. Green, M. Kato, K. Kiontke, A. Mortazavi, A. Seah, and B. Williams for comments on the manuscript. Some nematode strains used in this work were provided by the Caenorhabditis Genetics Center, which is funded by the NIH National Center for Research Resources (NCRR). Unpublished metazoan genomic sequences were generously provided by the DOE JGI and GeneDB. This work was supported by grants from DOE to B.J.W. and P.W.S., from NASA to B.J.W., from NIH to B.J.W., and from the HHMI, with which P.W.S. is an Investigator.

\section{References}

Aboobaker, A. and Blaxter, M. 2003. Hox gene evolution in nematodes: Novelty conserved. Curr. Opin. Genet. Dev. 13: 593-598.

Ahituv, N., Zhu, Y., Visel, A., Holt, A., Afzal, V., Pennacchio, L.A., and Rubin, E.M. 2007. Deletion of ultraconserved elements yields viable mice. PLoS Biol. 5: e234. doi: 10.1371/journal.pbio.0050234.

Ao, W., Gaudet, J., Kent, W.J., Muttumu, S., and Mango, S.E. 2004 Environmentally induced foregut remodeling by PHA-4/FoxA and DAF-12/NHR. Science 305: 1743-1746.

Bailey, T.L. and Elkan, C. 1994. Fitting a mixture model by expectation maximization to discover motifs in biopolymers. Proc. Int. Conf. Intell. Syst. Mol. Biol. 2: 28-36.

Baldwin, J.G., Frisse, L.M., Vida, J.T., Eddleman, C.D., and Thomas, W.K. 1997. An evolutionary framework for the study of developmental evolution in a set of nematodes related to Caenorhabditis elegans. Mol. Phylogenet. Evol. 8: 249-259.

Bejerano, G., Pheasant, M., Makunin, I., Stephen, S., Kent, W.J., Mattick, J.S., and Haussler, D. 2004. Ultraconserved elements in the human genome. Science 304: 1321-1325.

Bigelow, H.R., Wenick, A.S., Wong, A., and Hobert, O. 2004. CisOrtho: A program pipeline for genome-wide identification of transcription factor target genes using phylogenetic footprinting. $B M C$ Bioinformatics 5: 27. doi: 10.1186/1471-2105-5-27.

Birren, B., Mancino, V., and Shizuya, H. 1999. Bacterial artificial chromosomes. In Genome analysis: A laboratory manual (eds. B. Birren et al.), pp. 241-295. Cold Spring Harbor Laboratory Press, Cold Spring Harbor, NY.

Boffelli, D., Nobrega, M.A., and Rubin, E.M. 2004. Comparative genomics at the vertebrate extremes. Nat. Rev. Genet. 5: 456-465.

Brown, C.T. 2006. "Tackling the regulatory genome." Ph.D. thesis, California Institute of Technology, Pasadena.

Brown, C.T., Rust, A.G., Clarke, P.J., Pan, Z., Schilstra, M.J., De Buysscher, T., Griffin, G., Wold, B.J., Cameron, R.A., Davidson, E.H., et al. 2002. New computational approaches for analysis of cis-regulatory networks. Dev. Biol. 246: 86-102.

Brown, C.D., Johnson, D.S., and Sidow, A. 2007. Functional architecture and evolution of transcriptional elements that drive gene coexpression. Science 317: 1557-1560.

Brunschwig, K., Wittmann, C., Schnabel, R., Burglin, T.R., Tobler, H., and Muller, F. 1999. Anterior organization of the Caenorhabditis elegans embryo by the labial-like Hox gene ceh-13. Development 126: 1537-1546.

Bryne, J.C., Valen, E., Tang, M.H., Marstrand, T., Winther, O., da Piedade, I., Krogh, A., Lenhard, B., and Sandelin, A. 2007. JASPAR, the open access database of transcription factor-binding profiles: New content and tools in the 2008 update. Nucleic Acids Res. 36: D102-D106.

Burglin, T.R. and Ruvkun, G. 1993. The Caenorhabditis elegans homeobox gene cluster. Curr. Opin. Genet. Dev. 3: 615-620.

Cameron, R.A., Chow, S.H., Berney, K., Chiu, T.Y., Yuan, Q.A., Kramer, A., Helguero, A., Ransick, A., Yun, M., and Davidson, E.H. 2005. An evolutionary constraint: Strongly disfavored class of change in DNA sequence during divergence of cis-regulatory modules. Proc. Natl. Acad. Sci. 102: 11769-11774.

Chen, N., Mah, A., Blacque, O.E., Chu, J., Phgora, K., Bakhoum, M.W., Newbury, C.R., Khattra, J., Chan, S., Go, A., et al. 2006. Identification of ciliary and ciliopathy genes in Caenorhabditis elegans through comparative genomics. Genome Biol. 7: R126. doi: 10.1186/gb-2006-7-12-r126.

Cho, S., Jin, S.W., Cohen, A., and Ellis, R.E. 2004. A phylogeny of Caenorhabditis reveals frequent loss of introns during nematode evolution. Genome Res. 14: 1207-1220.

Clandinin, T.R., Katz, W.S., and Sternberg, P.W. 1997. Caenorhabditis elegans HOM-C genes regulate the response of vulval precursor cells to inductive signal. Dev. Biol. 182: $150-161$.

Clark, S.G., Chisholm, A.D., and Horvitz, H.R. 1993. Control of cell fates in the central body region of $C$. elegans by the homeobox gene lin-39. Cell 74: 43-55.

Cliften, P., Sudarsanam, P., Desikan, A., Fulton, L., Fulton, B., Majors, J., Waterston, R., Cohen, B.A., and Johnston, M. 2003. Finding functional features in Saccharomyces genomes by phylogenetic footprinting. Science 301: 71-76.

Colbert, H.A., Smith, T.L., and Bargmann, C.I. 1997. OSM-9, a novel protein with structural similarity to channels, is required for olfaction, mechanosensation, and olfactory adaptation in Caenorhabditis elegans. J. Neurosci. 17: 8259-8269.

Davidson, E.H. 2006. The regulatory genome: Gene regulatory networks in development and evolution. Academic Press, San Diego, CA.

Dickinson, W. 1991. The evolution of regulatory genes and patterns in Drosophila. Evol. Biol. 25: 127-173.

Eddy, S.R. 2005. A model of the statistical power of comparative genome sequence analysis. PLoS Biol. 3: e10. doi: 10.1371/journal.pbio.0030010.

Engström, P.G., Ho Sui, S.J., Drivenes, O., Becker, T.S., and Lenhard, B. 2007. Genomic regulatory blocks underlie extensive microsynteny conservation in insects. Genome Res. 17: 1898-1908.

Etchberger, J.F. and Hobert, O. 2008. Vector-free DNA constructs improve transgene expression in C. elegans. Nat. Methods 5: 3. doi: 10.1038/nmeth0108-3.

Etchberger, J.F., Lorch, A., Sleumer, M.C., Zapf, R., Jones, S.J., Marra, M.A., Holt, R.A., Moerman, D.G., and Hobert, O. 2007. The molecular signature and cis-regulatory architecture of a C. elegans gustatory neuron. Genes \& Dev. 21: 1653-1674.

Frasch, M., Chen, X., and Lufkin, T. 1995. Evolutionary-conserved enhancers direct region-specific expression of the murine Hoxa-1

\section{Genome Research}


and Hoxa-2 loci in both mice and Drosophila. Development 121: 957-974.

Garcia-Fernandez, J. 2005. The genesis and evolution of homeobox gene clusters. Nat. Rev. Genet. 6: 881-892.

Gaudet, J., Muttumu, S., Horner, M., and Mango, S.E. 2004 Whole-genome analysis of temporal gene expression during foregut development. PLoS Biol. 2: e352. doi: 10.1371/journal.pbio.0020352.

Grant, K., Hanna-Rose, W., and Han, M. 2000. sem-4 promotes vulval cell-fate determination in Caenorhabditis elegans through regulation of lin-39 Hox. Dev. Biol. 224: 496-506.

Graustein, A., Gaspar, J.M., Walters, J.R., and Palopoli, M.F. 2002. Levels of DNA polymorphism vary with mating system in the nematode genus Caenorhabditis. Genetics 161: 99-107.

Haerry, T.E. and Gehring, W.J. 1997. A conserved cluster of homeodomain binding sites in the mouse Hoxa-4 intron functions in Drosophila embryos as an enhancer that is directly regulated by Ultrabithorax. Dev. Biol. 186: 1-15.

Hillier, L.W., Coulson, A., Murray, J.I., Bao, Z., Sulston, J.E., and Waterston, R.H. 2005. Genomics in C. elegans: So many genes, such a little worm. Genome Res. 15: 1651-1660.

Hobert, O. 2002. PCR fusion-based approach to create reporter gene constructs for expression analysis in transgenic C. elegans. Biotechniques 32: 728-730.

Kellis, M., Patterson, N., Endrizzi, M., Birren, B., and Lander, E.S. 2003. Sequencing and comparison of yeast species to identify genes and regulatory elements. Nature 423: 241-254.

Kelly, W.G., Xu, S., Montgomery, M.K., and Fire, A. 1997. Distinct requirements for somatic and germline expression of a generally expressed Caenorhabditis elegans gene. Genetics 146: 227-238.

Kimble, J. and Hirsh, D. 1979. The postembryonic cell lineages of the hermaphrodite and male gonads in Caenorhabditis elegans. Dev. Biol. 70: $396-417$.

Kiontke, K., Hironaka, M., and Sudhaus, W. 2002. Description of Caenorhabditis japonica n. sp. (Nematoda: Rhabditida) associated with the burrower bug Parastrachia japonensis (Heteroptera: Cydnidae) in Japan. Nematology 4: 933-941.

Kiontke, K., Gavin, N.P., Raynes, Y., Roehrig, C., Piano, F., and Fitch, D.H. 2004. Caenorhabditis phylogeny predicts convergence of hermaphroditism and extensive intron loss. Proc. Natl. Acad. Sci. 101: 9003-9008.

Kiontke, K., Barriere, A., Kolotuev, I., Podbilewicz, B., Sommer, R., Fitch, D.H., and Felix, M.A. 2007. Trends, stasis, and drift in the evolution of nematode vulva development. Curr. Biol. 17: 1925-1937.

Korf, I., Yandell, M., and Bedell, J. 2003. BLAST. O'Reilly, Sebastopol, CA.

Krause, M., Harrison, S.W., Xu, S.Q., Chen, L., and Fire, A. 1994. Elements regulating cell- and stage-specific expression of the C. elegans MyoD family homolog hlh-1. Dev. Biol. 166: 133-148.

Krek, A., Grün, D., Poy, M.N., Wolf, R., Rosenberg, L., Epstein, E.J., MacMenamin, P., da Piedade, I., Gunsalus, K.C., Stoffel, M., et al. 2005. Combinatorial microRNA target predictions. Nat. Genet. 37: 495-500.

Lemons, D. and McGinnis, W. 2006. Genomic evolution of Hox gene clusters. Science 313: 1918-1922.

Li, L., Stoeckert Jr., C.J., and Roos, D.S. 2003. OrthoMCL: Identification of ortholog groups for eukaryotic genomes. Genome Res. 13: 2178-2189.

Li, L., Zhu, Q., He, X., Sinha, S., and Halfon, M.S. 2007. Large-scale analysis of transcriptional cis-regulatory modules reveals both common features and distinct subclasses. Genome Biol. 8: R101. doi: 10.1186/gb-2007-8-6-r101.

Lim, L.P., Lau, N.C., Weinstein, E.G., Abdelhakim, A., Yekta, S., Rhoades, M.W., Burge, C.B., and Bartel, D.P. 2003. The microRNAs of Caenorhabditis elegans. Genes \& Dev. 17: 991-1008.

Malicki, J., Cianetti, L.C., Peschle, C., and McGinnis, W. 1992. A human HOX4B regulatory element provides head-specific expression in Drosophila embryos. Nature 358: 345-347.

Maloof, J.N. and Kenyon, C. 1998. The Hox gene lin-39 is required during C. elegans vulval induction to select the outcome of Ras signaling. Development 125: 181-190.

McGaughey, D.M., Vinton, R.M., Huynh, J., Al-Saif, A., Beer, M.A., and McCallion, A.S. 2008. Metrics of sequence constraint overlook regulatory sequences in an exhaustive analysis at phox $2 b$. Genome Res. 18: 252-260.

McGhee, J.D., Sleumer, M.C., Bilenky, M., Wong, K., McKay, S.J., Goszczynski, B., Tian, H., Krich, N.D., Khattra, J., Holt, R.A., et al. 2007. The ELT-2 GATA-factor and the global regulation of transcription in the C. elegans intestine. Dev. Biol. 302: 627-645.

McKay, S.J., Johnsen, R., Khattra, J., Asano, J., Baillie, D.L., Chan, S., Dube, N., Fang, L., Goszczynski, B., Ha, E., et al. 2003. Gene expression profiling of cells, tissues, and developmental stages of the nematode C. elegans. Cold Spring Harb. Symp. Quant. Biol. 68: $159-169$.

Mello, C. and Fire, A. 1995. DNA transformation. Methods Cell Biol. 48: 451-482.

Mortazavi, A., Leeper Thompson, E.C., Garcia, S.T., Myers, R.M., and Wold, B. 2006. Comparative genomics modeling of the NRSF/REST repressor network: From single conserved sites to genome-wide repertoire. Genome Res. 16: 1208-1221.

Okkema, P.G., Harrison, S.W., Plunger, V., Aryana, A., and Fire, A. 1993 Sequence requirements for myosin gene expression and regulation in Caenorhabditis elegans. Genetics 135: 385-404.

Olson, S. 2002. EMBOSS opens up sequence analysis. European Molecular Biology Open Software Suite. Bioinformatics 3: 87-91.

Ovcharenko, I., Loots, G.G., Nobrega, M.A., Hardison, R.C., Miller, W. and Stubbs, L. 2005. Evolution and functional classification of vertebrate gene deserts. Genome Res. 15: 137-145.

Pauli, F., Liu, Y., Kim, Y.A., Chen, P.J., and Kim, S.K. 2006. Chromosomal clustering and GATA transcriptional regulation of intestine-expressed genes in C. elegans. Development 133: 287-295.

Pennacchio, L.A., Ahituv, N., Moses, A.M., Prabhakar, S., Nobrega, M.A. Shoukry, M., Minovitsky, S., Dubchak, I., Holt, A., Lewis, K.D., et al. 2006. In vivo enhancer analysis of human conserved non-coding sequences. Nature 444: 499-502.

Popperl, H., Bienz, M., Studer, M., Chan, S.K., Aparicio, S., Brenner, S., Mann, R.S., and Krumlauf, R. 1995. Segmental expression of Hoxb-1 is controlled by a highly conserved autoregulatory loop dependent upon exd/pbx. Cell 81: 1031-1042.

Ruvinsky, I. and Ruvkun, G. 2003. Functional tests of enhancer conservation between distantly related species. Development 130: 5133-5142.

Sandelin, A., Alkema, W., Engstrom, P., Wasserman, W.W., and Lenhard, B. 2004. JASPAR: An open-access database for eukaryotic transcription factor binding profiles. Nucleic Acids Res. 32: D91-D94.

Sinha, S., Schroeder, M.D., Unnerstall, U., Gaul, U., and Siggia, E.D. 2004. Cross-species comparison significantly improves genome-wide prediction of cis-regulatory modules in Drosophila. BMC Bioinformatics 5: 129. doi: 10.1186/1471-2105-5-129.

Stajich, J.E., Block, D., Boulez, K., Brenner, S.E., Chervitz, S.A., Dagdigian, C., Fuellen, G., Gilbert, J.G., Korf, I., Lapp, H., et al. 2002. The Bioperl toolkit: Perl modules for the life sciences. Genome Res. 12: 1611-1618.

Stark, A., Lin, M.F., Kheradpour, P., Pedersen, J.S., Parts, L., Carlson, J.W., Crosby, M.A., Rasmussen, M.D., Roy, S., Deoras, A.N., et al. 2007. Discovery of functional elements in 12 Drosophila genomes using evolutionary signatures. Nature 450: 219-232.

Sternberg, P.W. 2005. Vulval development. In WormBook (eds. The C. elegans Research Community). doi: 10.1895/wormbook.1.6.1, http://www.wormbook.org.

Stone, E.A., Cooper, G.M., and Sidow, A. 2005. Trade-offs in detecting evolutionarily constrained sequence by comparative genomics. Annu. Rev. Genomics Hum. Genet. 6: 143-164.

Stothard, P. and Pilgrim, D. 2006. Conspecific and interspecific interactions between the FEM-2 and the FEM-3 sex-determining proteins despite rapid sequence divergence. J. Mol. Evol. 62: 281-291.

Stoyanov, C.N., Fleischmann, M., Suzuki, Y., Tapparel, N., Gautron, F., Streit, A., Wood, W.B., and Muller, F. 2003. Expression of the C. elegans labial orthologue ceh-13 during male tail morphogenesis. Dev. Biol. 259: 137-149.

Streit, A., Kohler, R., Marty, T., Belfiore, M., Takacs-Vellai, K., Vigano, M.A., Schnabel, R., Affolter, M., and Muller, F. 2002. Conserved regulation of the Caenorhabditis elegans labial/Hox1 gene ceh-13. Dev. Biol. 242: 96-108.

Sudhaus, W. and Kiontke, K. 1996. Phylogeny of Rhabditis subgenus Caenorhabditis (Rhabditidae, Nematoda). J. Zoo. Syst. Evol. 34: 217-233.

Sudhaus, W. and Kiontke, K. 2007. Comparison of the cryptic nematode species Caenorhabditis brenneri sp. $\mathrm{n}$. and C. remanei (Nematoda: Rhabditidae) with the stem species pattern of the Caenorhabditis elegans group. Zootaxa 1456: 45-62.

Sulston, J. and Hodgkin, J. 1988. Methods. In The nematode Caenorhabditis elegans (ed. W.B. Wood), pp. 587-606. Cold Spring Harbor Laboratory, Cold Spring Harbor, NY.

Sulston, J.E. and Horvitz, H.R. 1977. Post-embryonic cell lineages of the nematode, Caenorhabditis elegans. Dev. Biol. 56: 110-156.

Tagle, D.A., Koop, B.F., Goodman, M., Slightom, J.L., Hess, D.L., and Jones, R.T. 1988. Embryonic and globin genes of a prosimian primate (Galago crassicaudatus). Nucleotide and amino acid sequences, developmental regulation and phylogenetic footprints J. Mol. Biol. 203: 439-455.

Tompa, M., Li, N., Bailey, T.L., Church, G.M., De Moor, B., Eskin, E., 


\section{Kuntz et al.}

Favorov, A.V., Frith, M.C., Fu, Y., Kent, W.J., et al. 2005. Assessing computational tools for the discovery of transcription factor binding sites. Nat. Biotechnol. 23: 137-144.

Wagmaister, J.A., Miley, G.R., Morris, C.A., Gleason, J.E., Miller, L.M., Kornfeld, K., and Eisenmann, D.M. 2006. Identification of cis-regulatory elements from the C. elegans Hox gene lin-39 required for embryonic expression and for regulation by the transcription factors LIN-1, LIN-31 and LIN-39. Dev. Biol. 297: 550-565.

Wang, X. and Chamberlin, H.M. 2004. Evolutionary innovation of the excretory system in Caenorhabditis elegans. Nat. Genet. 36: 231-232.

Wang, T. and Stormo, G.D. 2003. Combining phylogenetic data with co-regulated genes to identify regulatory motifs. Bioinformatics 19: 2369-2380.

Wang, B.B., Muller-Immergluck, M.M., Austin, J., Robinson, N.T., Chisholm, A., and Kenyon, C. 1993. A homeotic gene cluster patterns the anteroposterior body axis of C. elegans. Cell 74: 29-42.

Wei, C., Lamesch, P., Arumugam, M., Rosenberg, J., Hu, P., Vidal, M., and Brent, M.R. 2005. Closing in on the C. elegans ORFeome by cloning TWINSCAN predictions. GenomeRes. 15: 577-582.

Wenick, A.S. and Hobert, O. 2004. Genomic cis-regulatory architecture and trans-acting regulators of a single interneuron-specific gene battery in C. elegans. Dev. Cell 6: 757-770.

Wittmann, C., Bossinger, O., Goldstein, B., Fleischmann, M., Kohler, R.,
Brunschwig, K., Tobler, H., and Muller, F. 1997. The expression of the C. elegans labial-like Hox gene ceh-13 during early embryogenesis relies on cell fate and on anteroposterior cell polarity. Development 124: $4193-4200$.

Woolfe, A., Goodson, M., Goode, D.K., Snell, P., McEwen, G.K., Vavouri, T., Smith, S.F., North, P., Callaway, H., Kelly, K., et al. 2005. Highly conserved non-coding sequences are associated with vertebrate development. PLoS Biol. 3: e7. doi: 10.1371/journal.pbio.0030007.

Xie, X., Lu, J., Kulbokas, E.J., Golub, T.R., Mootha, V., Lindblad-Toh, K., Lander, E.S., and Kellis, M. 2005. Systematic discovery of regulatory motifs in human promoters and 3' UTRs by comparison of several mammals. Nature 434: 338-345.

Xie, X., Mikkelsen, T.S., Gnirke, A., Lindblad-Toh, K., Kellis, M., and Lander, E.S. 2007. Systematic discovery of regulatory motifs in conserved regions of the human genome, including thousands of CTCF insulator sites. Proc. Natl. Acad. Sci. 104: 7145-7150.

Zhao, G., Schriefer, L.A., and Stormo, G.D. 2007. Identification of muscle-specific regulatory modules in Caenorhabditis elegans. Genome Res. 17: 348-357.

Received August 26, 2008; accepted in revised form September 17, 2008. 


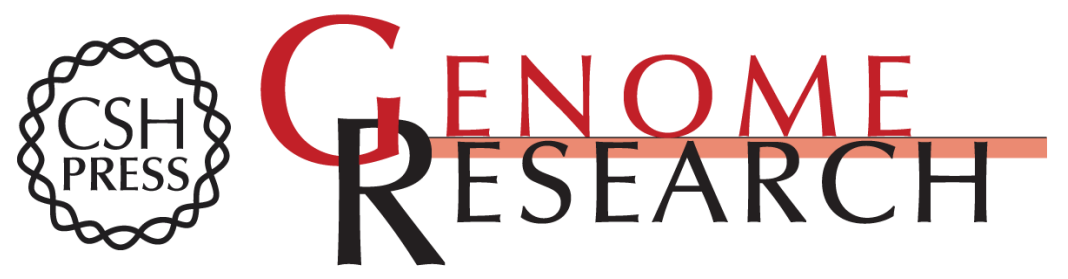

\section{Multigenome DNA sequence conservation identifies Hox cis -regulatory elements}

Steven G. Kuntz, Erich M. Schwarz, John A. DeModena, et al.

Genome Res. 2008 18: 1955-1968 originally published online November 3, 2008

Access the most recent version at doi:10.1101/gr.085472.108

Supplemental Material

References

License

Email Alerting Service
http://genome.cshlp.org/content/suppl/2008/11/06/gr.085472.108.DC1

This article cites 83 articles, 31 of which can be accessed free at: http://genome.cshlp.org/content/18/12/1955.full.html\#ref-list-1

Receive free email alerts when new articles cite this article - sign up in the box at the top right corner of the article or click here.

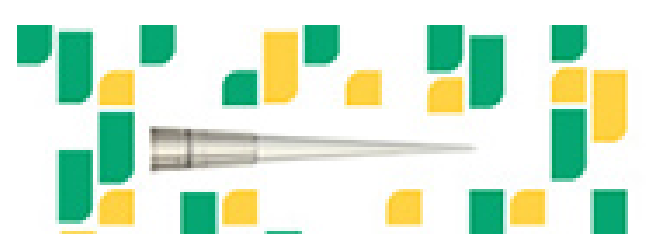

Focused on your science.

Jפగ

SCIENTIFIC

suos or seisnes

To subscribe to Genome Research go to:

https://genome.cshlp.org/subscriptions 